\title{
Physics Base \& Conceptual Views Complex of Ball Lightning
}

\author{
Anton V. Pinchuk ${ }^{1}$, Vladimir A. Pinchuk ${ }^{2}$ \\ ${ }^{1}$ Scientific Technical Center "Protei", Saint_Petersburg, Russia \\ ${ }^{2}$ The Baltic state technical university, Saint_Petersburg, Russia \\ E-mail:vap@vp7550.spb.edu \\ Received June 23, 2010; revised August 27, 2010; accepted September 16, 2010
}

\begin{abstract}
The complex of conceptual views about ball lightning (BL) as containing surplus electrical charge quasistationary material foundation with distinctions between levels of translation temperature and exciting temperatures of chemical bonds and electron energy states has been offered and is substantiated in the paper. The being formed complex is grounded upon overstepping the limits of generally accepted physical base and is aimed at all-round substantiation of jointly observed in nature manifestations of BL. Within the limits of the being formed complex the possibility of localization of ball lightning in airspace is confirmed. The nature of the one's radiate capacity is substantiated. The role of environment as source that feeds BL with energy is established. Power supply mechanisms, conditions and peculiarities of BL's characteristics reproduction through lifetime are specified. The formations channels of energy resources of BL are turned out. Levels of BL's energy potentialities, both permissibility proper and wide diapason of variation of the ones (multi-faces of ball lightning) as well as the capability of BL to overcome any barriers from dielectric materials by means of penetration through inanes in body of barriers and then to restore the one's characteristic (octopus's effect) are substantiated. Adequacy of being formulated conceptual views as a whole is confirmed.
\end{abstract}

Keywords: Ball Lightning, Natural Anomaly

\section{Introduction}

In 1838 year Fransuar Arago-the member of French Science Academy published the article with generalization of several views of Ball Lightning (BL). Since then and up till now the substantiation problem of BL's nature is in the attention centre of scientists of the all world. The numerous works (Barry J., Dmitriev M. T., Elliott lo., Singer S., Silberg P., Shabanov G. D., Sokolovski B. Yu. Smirnov B. M., Stakhanov I. P., ...) directed onto both the generalization of ball lightning observations and making-up of a common ideas of the ones are known.

Among important results of their joint work the portrait of Ball Lightning, having been formulated by generalization of numerous observations of $\mathrm{BL}$ in the nature, should be mentioned above all. The one can be presented, for example, by the next means.

The ball lightning $(B L)$ in itself is of nature origin, being displaying with energy radiation into environment a material formation, localized in airspace as rotation ellipsoid or even in the similar to spherical shape, the one being able to move, primary motion directions of that aren't determined, however, by gravitation and/or air dynamic forces and lifetime of that, accordingly with observations, is at least tens of second. Characteristics of BL, being only insignificantly changed or even kept practically constant in lifetime, don't register seemingly evident reduction of energy resources of ball lightning during time of one's existence in connection with radiation energy to environment. What is more, the disappearance (disintegration) proper of BL is accompanied as a rule with an additional energy discharge into environment (frequently considerably exceeds quasi-stationary level of energy radiation). Multi-faces being manifested first of all by energy levels differences being discharged into environment with BLs at the disappearance period under seemingly the same characteristics of the ones' quasi-stationary state typical to $B L$.

At last "Octopus's effect", that is capability of BL to overcome different barriers from dielectric materials (window-frame, for example) by means of penetration through inanes in the ones' body (hollows, cracks, splits) 
with essentially lesser sizes, concerning to dimensions of $B L$ and then to restore (reconstruct) in essence initial shape and characteristics is peculiar to BL too.

However up to now, even after the lapse of about two century, the generally accepted conception of both nature and jointly fixed characteristics of BL on the base of universally recognized physical views is not turned out yet. What's more the true sensation of pessimism in estimations of principal possibilities to interpret the BL nature on this basis is felt.

It's undoubtedly, that the being settled situation shows first of all to exceptional complexity of BL as the investtigation object. At the same time, it unquestionably notes too towards the principal insufficiency of resolving power of being used investigation methods formed in these cases in the framework of traditional physical restrictions (i.e. towards imperfection of the physical basis as itself).

It is interesting to note in this connection that the science history is rich with examples when the one or other problem, occurring itself interest for investigations and being very important just as from practical so from science standpoint, within of generally adopted physical limits is kept unsolved and only going out of these limits is way to solve the one. In other words there is reason so to think that the Ball Lightning in the greatest degree is in keeping with these features.

The attempts to work out the complex substantiation of both the nature and manifestation of BL on the basis of overstepping the limits of traditional physical restricttions, earlier unknown Internal Energy Equilibrium Conditions (IEEC-between energy states with different nature) of material media are known too [1-13].

Admittedly, the developed views of BL so far do not obtain of due expansion and of necessary public recognition (in that number owing to the being kept till now doubts about substantiations of proper IEEC) and demand so additional explanation and progress.

Having been formed situation and determined in essence expediency and purposefulness of this work. The paper is based upon materials of [1-13] and, at the same time, is aimed onto generalization and further logical progress of the ones.

\section{Strategy Forming}

\subsection{Initial Views}

On being formed of initial views of BL we will take into account only common, beyond all doubt observed of ball lightning's characteristics.

It must be admitted so that BLs exist and are spring up under conditions of developed electro-static fields. They are moved somewhere else and are observed as localized in the airspace material formations having form similar to sphere. The prevailing transferences directions of ball lightning in airspace are not determined by the both gravitation and/or forces of aerodynamic origin ${ }^{1}$. BL's lifetime may achieve at least to several tens seconds.

The BL occurrence, as a rule, is accompanied by energy radiation into environment (thermodynamically opened system) that is watched in the range of visible spectrum part in that number. As this takes place, characteristics of BL's radiation are changed insignificantly or even are kept practically an invariable. What is more, disintegration of ball lightning often is accompanied with extra energy escape into environment. The multi-faces, revealed by considerable incompatibility of specific energy resources under seemingly equal or at least comparable circumstances, else is peculiar to BL (see, for example, $[15,16])$. Octopus's effect-capability to overcome different barriers from dielectric materials by means of penetration through inanes in the body of barrier with essentially lesser, concerning to dimensions of BL, sizes and then to restore (reconstruct) in essence the ones' initial shape and properties characteristic is characteristic of Ball Lightning at last. The enumerated common properties with a great degree of trustworthiness are suited to the real manifestations of balls lightning. However, up till now the possibilities to realize the medium states with noted characteristics intake, in the context of generally accounted physics-chemistry views, are retained beyond verge of explainable ones.

\subsection{Basic Assumption}

In evaluating of prospects for refinements of BL's nature in the framework of [1-13] the attention was being given first of all to the established by observations fact that the BL-medium contains surplus (uncompensated) electric charge.

As an assumption, in connection with this, it was presupposed that

"the observed characteristics of BL could be substantiated, say, by the existence of traditionally not accounted (earlier unknown) peculiarities of internal energy equilibrium conditions (IEEC-between energy states with physical nature's different kinds) for material formations with surplus electric charge (AQN- or aquasineutral formations) as determining, in that case by the results of inevitably being exited relaxation of $A Q N$ formations on interacting with environment, and arising and subsequent conservation in time (reproduction) of

\footnotetext{
${ }^{1}$ The marked indicates so that $\mathrm{BL}$ is subjected to force influences of electric nature, i.e. keeps an uncompensated (surplus) electric charge. The last is confirmed else by known theoretical investigations [14].
} 
medium (in the AQN-formation's composition) with characteristics that differ from the environment and are in keeping to $B L$ ".

The possibilities to build on this base the physics-chemic BL's model will be estimate drawing away our attention from both the composition and the nature of BL's formation mechanisms as the subjects of specific interest as itself.

\section{The Internal Energy Equilibrium Conditions (IEEC)}

\subsection{Ionization Equilibrium Conditions}

Let's estimate for the first time the possibly arising, in connection with presence in medium's composition of surplus charge to fit the being expressed assumption, the peculiarities of the medium's inner ionization equilibrium.

\subsubsection{Small Disturbances Method}

As applied to $p, T=$ Const let us separate out now some volume of medium containing neutral (a), electron (e) and ion (i) components. Let us account that the separated so volume is bounded, for instance, by sphere of radius $R_{b l}$ and is thermodynamically opened system itself with variable particles number and description of the one conforms to three-liquid model ideas [17].

We'll be take, as state parameters, the ionization degree

$$
\alpha^{*}=\frac{p_{i}^{*}}{p_{a}^{(0)}}=\frac{n_{i}^{*} k T}{n_{a}{ }^{(0)} k T}=\frac{n_{i}^{*}}{n_{a}^{(0)}}
$$

and relative content of uncompensated electron component ${ }^{2}$

$$
\psi=\frac{p_{e}^{*}-p_{i}^{*}}{p_{i}^{*}}=\frac{\left(n_{e}^{*}-n_{i}^{*}\right) k T}{n_{i}^{*} k T}=\frac{n_{e}^{*}-n_{i}^{*}}{n_{i}^{*}} .
$$

Concentrations of components in medium's composition in that case will be conform to relations

$$
\begin{aligned}
& n_{i}{ }^{*}=\alpha^{*} n_{a}{ }^{(o)} ; \quad n_{e}{ }^{*}=\alpha^{*}(1+\psi) n_{a}{ }^{(0)} ; \quad n_{a}=\left(1-\alpha^{*}\right) n_{a}{ }^{(0)} ; \\
& n_{\Sigma}=\left[1+\alpha^{*}(1+\psi)\right] n_{a}{ }^{(0)}
\end{aligned}
$$

Give up advantage to thermodynamic method as not leaning upon any ideas of substance atomic-molecular structure and being essentially phenomenological. Assume that by external in relation to sphere actions an excess electric charge is inputted and at a later time is retained in the composition of separated so medium volume.

Accordingly generally adopted ideas inner equilibrium of separated system should be in conformity with the

${ }^{2}$ Here and below with $« * »$ the parameters of media that contains surplus charge are noted. minimum of its thermodynamic isobaric-isothermal potential " $\Phi$ " and, for $p, T=$ Const, will be determined in that case by equation

$$
\begin{aligned}
& \delta \Phi=\sum_{1}^{J}\left(\frac{\partial \Phi}{\partial N_{j}}\right)_{\bar{p}, \bar{T}}\left(\frac{\partial N_{j}}{\partial \psi}\right)_{\bar{\alpha}^{*}} \delta \psi \\
& +\sum_{1}^{j}\left(\frac{\partial \Phi}{\partial N_{j}}\right)_{\bar{p}, \bar{T}}\left(\frac{\partial N_{j}}{\partial \alpha^{*}}\right)_{\bar{\psi}} \delta \alpha^{*}=\sum_{1}^{j} \mu_{j}\left(\frac{\partial N_{j}}{\partial \psi}\right)_{\bar{\alpha}^{*}} \delta \psi \\
& +\sum_{1}^{j} \mu_{j}\left(\frac{\partial N_{j}}{\partial \alpha^{*}}\right)_{\bar{\psi}} \delta \alpha^{*}=0 .
\end{aligned}
$$

Here: $\mu_{j}$-chemical potential of $j$-'s media component $(j=a, e, i)$.

Draw attention to the fact that under conditions when the charge within the sphere is constant

$\left(\Delta n_{q}=n_{e}{ }^{*}-n_{i}{ }^{*}=\right.$ Const $)$ parameter

$$
\psi=\frac{n_{e}{ }^{*}-n_{i}{ }^{*}}{n_{i}{ }^{*}}=\frac{\Delta n_{q}}{\alpha n_{a}{ }^{(0)}}=f\left(\alpha^{*}\right)
$$

and first term of right part in equation (3) is identically equal to zero. Equation (3) is transformed so to expression

$$
\sum_{1}^{j} \mu_{j}\left(\frac{\partial N_{j}}{\partial \alpha^{*}}\right)_{\bar{\psi}} \delta \alpha^{*}=\sum_{1}^{j} \mu_{j} \delta N_{j}=0
$$

and may be used for detecting of correlations between states parameters of media plasma foundations corresponding to inner energy equilibrium.

For the material system of examined composition the equation (5) is rewrite in

$$
\mu_{i} \delta N_{i}+\mu_{e} \delta N_{e}+\mu_{a} \delta N_{a}=0
$$

and, with account of correlations between moles numbers variations $\delta N_{i} \approx \delta n_{i}=n_{a}{ }^{(0)} \delta \alpha$;

$$
\delta N_{e} \approx \delta n_{e}=(1+\psi) n_{a}{ }^{(0)} \delta \alpha ; \quad \delta N_{a} \approx \delta n_{a}=-n_{a}{ }^{(0)} \delta \alpha
$$

in accordance with (1). (2), is reduced to relationship

$$
\mu_{i}+\mu_{e}(1+\psi)-\mu_{a}=0
$$

For single-atom gas

$$
\begin{aligned}
& \mu_{j}=R T\left[\ln p_{j}-\ln \frac{g_{j}\left(2 \pi m_{j}\right)^{3 / 2}(k T)^{5 / 2}}{h^{3}}\right]+ \\
& +\varepsilon_{j}{ }^{0}+e Z_{j} N_{A} \Omega, \quad Z_{j}=\left\{\begin{array}{l}
1, \text { if } j=i, \\
0, \text { if } j=a, \\
-1, \text { if } j=e .
\end{array}\right.
\end{aligned}
$$

Expression (7) is converted into 


$$
\begin{aligned}
& \prod_{j} p_{j}^{c_{j}}=\prod_{j}\left[g_{j} \frac{\left(2 \pi m_{j}\right)^{3 / 2}(k T)^{5 / 2}}{h^{3}}\right]^{c_{j}} \times \\
& \times \exp \left(-e \frac{V-\psi \Omega}{k T}\right), \quad c_{j}=\left\{\begin{array}{c}
1, \text { if } j=i, \\
(1+\psi), \text { if } j=e, \\
-1, \text { if } j=a .
\end{array}\right.
\end{aligned}
$$

and, on the neglect of 2-order infinitesimal members, corresponds to any from equations:

$$
\begin{aligned}
& \frac{p_{i}^{*} p_{e}^{*(1+\psi)}}{p_{a}}=\frac{g_{i}}{g_{a}}\left[g_{e} \frac{\left(2 \pi m_{e}\right)^{3 / 2}(k T)^{5 / 2}}{h^{3}}\right]^{(1+\psi)} \times \\
& \times \exp \left(-e \frac{V-\psi \Omega}{k T}\right), \\
& \frac{n_{i}^{*} n_{e}^{*(1+\psi)}}{n_{a}}=\frac{g_{i}}{g_{a}}\left[g_{e} \frac{\left(2 \pi m_{e} k T\right)^{3 / 2}}{h^{3}}\right]^{(1+\psi)} \times \\
& \times \exp \left(-e \frac{V-\psi \Omega}{k T}\right)
\end{aligned}
$$

or

$$
\begin{aligned}
& \frac{\alpha^{*(2+\psi)}(1+\psi)^{(1+\psi)}}{\left(1-\alpha^{*}\right)\left[1+\alpha^{*}(1+\psi)\right]^{(1+\psi)}}=\frac{1}{P_{\Sigma}^{(1+\psi)}} \frac{g_{i}}{g_{a}} \times \\
& \times\left[g_{e} \frac{\left(2 \pi m_{e}\right)^{3 / 2}(k T)^{5 / 2}}{h^{3}}\right]^{(1+\psi)} \exp \left(-e \frac{V-\psi \Omega}{k T}\right)
\end{aligned}
$$

Here: $V$-media ionization potential determined in the ordinary way;

$\Omega$-remote coulomb interaction potential (potentials difference stipulating electrical action onto charge). More detailed interpretation of its physical sense will be given lower.

\subsubsection{Ionization Equilibrium as Reaction onto Disturbance}

Assume as previously that some quantity of the same charged particles are brought in (taken out from) the considered volume owing to the system is moved up (and at a later time exists) into the state with uncompensated electric charge. It is obvious that charge introduction into a system is equivalent to a work performance under the one and causes so violation of inner energy equilibrium of the system. With account of Le C hatelier Principle the last should being reestablished by inevitably arising in these conditions system reaction, suppose in the $A \Leftrightarrow A^{+}+e$ kind.

If a work has been done under the system by external actions is $\delta L_{1}=\mu_{e} \delta N_{e_{1}}$ and a change of the system's thermodynamic potential was stipulated on the system with external actions is $\delta \Phi=\mu_{i} \delta N_{i}+\mu_{e} \delta N_{e}+\mu_{a} \delta N_{a}$ then being anew settled equilibrium must corresponds to To condition

$$
\mu_{i} \delta N_{i}+\mu_{e} \delta N_{e}+\mu_{a} \delta N_{a}=-\mu_{e} \delta N_{e_{1}} .
$$

It is obvious that $\delta N_{i}=\delta N_{e}=-\delta N_{a}$ and (13) is presented in the kind

$$
\mu_{i}+\mu_{e}-\mu_{a}=-\mu_{e} \frac{\delta N_{e_{1}}}{\delta N_{i}} .
$$

The ratio $\delta N_{e_{1}} / \delta N_{i}=\delta N_{e_{1}} / \delta N_{e}$ determines the molar part of excess charge carriers per ion component mole and corresponds in being again settled gas medium composition to system's state parameter $\psi$ according (2). The equation (14) is truly kept to the relations (9)(12), which proves the statement.

\subsection{Common Character of Inner Equilibrium}

In deducing on (9)-(12) we assumed that inner equilibrium disturbance by carrying in system's composition of surplus charge is necessarily suppressed with reaction of $A \Leftrightarrow A^{+}+e$ kind. However the noted is not having one.

Indeed, "from all possible steady states of thermodynamic system being allowed with border conditions of the law of mass transfer and conservation along with $2^{\text {nd }}$ law of thermodynamic the state with minimum production of entropy is realized" [18], to say, that which is priority one.

Let us assume that priority reaction formed in the subsystem as response onto disturbance in connection with carrying in medium composition of surplus charge is reaction of $A+B \Leftrightarrow A B$ shape. If the work was done in the case under system by external actions is $\delta L_{1}=\mu_{e} \delta N_{e_{1}}$ that being anew-formed equilibrium will correspond to the condition

$$
\mu_{A B} \delta N_{A B}+\mu_{A} \delta N_{A}+\mu_{B} \delta N_{B}=-\mu_{e} \delta N_{e_{1}} .
$$

Take into account that the relation molar contribution into a relaxation reaction of electron component $\psi=\delta N_{e_{1}} / \delta N_{A B}$ (that in full measure correlates with physical interpretation of parameter according to expression (2)) and $\delta N_{A B}=-\delta N_{A}=-\delta N_{B}$. By this means in neglecting of 2-order infinitesimal quantities the expression (15) may be written in the form

$$
\begin{aligned}
& \prod_{j} p_{j}^{c_{j}}=\prod_{j} K(T)_{j}^{c_{j}} \exp \left(-\frac{\varepsilon_{a c t}-e \psi \Omega}{k T}\right), \\
& c_{j}=\left\{\begin{array}{c}
1, \quad \text { if } j=A B, \\
\psi, \text { if } j=e, \\
-1, \text { if } j=A, B .
\end{array}\right.
\end{aligned}
$$

Here: $\varepsilon_{\text {act }}-$ association reaction's activation energy 
(or any other one's that in frame of specifically arising conditions in the system is priority one) being determined in essence by generally accepted method.

\subsection{Equilibrium Inner Peculiarities}

The expressions (9)-(12), (16) determine so Inner Energy Equilibrium Conditions (IEEC) in material media compositions in that number with account of possible presence of surplus electric charge in the ones composition. From analogues of generally accepted form the lasts are distinguished essentially with only presence in exponent indexes of additional term $e \psi \Omega$.

Concrete character of IEEC accordingly (9)-(12), (16) indicates so that, as applied to localized AQN-foundations temperature $T$ as traditionally estimated parameter directly determines equilibrium populations of energy states only with "mechanical" nature (translation, oscillating, rotatory ones).

If in the framework of traditional description and with account of (9)-(12), (18) $T_{e x c}{ }^{*}$-the excitation temperatures of chemical bonds and electron energy states of medium then their correlations with $T$-the excitation temperature of mechanical energy states (after referred to as translation one) to be determined by equation

$$
T_{e x c}^{*}=T \frac{\varepsilon_{j}}{\varepsilon_{j}-e \psi \Omega}
$$

Corresponding to IEEC the function of population distribution of chemical bonds and electron energy states will be correspond with relation

$$
N_{\varepsilon_{j}}^{*} \approx \exp \left(-\frac{\varepsilon_{j}-e \psi \Omega}{k T}\right)
$$

By IEEC, the population distribution function of chemical bonds and electron energy states in medium of AQN-foundations (directly since formation moment of the ones) is necessarily transformed (or at least is put to the deformation) to the form (18).

In other words IEEC in the form of (9)-(12), (16) really point to existence of traditionally not accounted (unknown) control channel over material media states (chargeous control channel), where electrical charge plays a role of control factor.

Draw attention that the transfer of the formed AQNformation in new corresponding to (9)-(12), (16)-(18) state (one's relaxation) is providedwith own energy-resources of AQN-formation. The AQN-formation during relaxation towards IEEC makes so some negative work. If work done by the medium of AQN-formation in this case is $-L=q \Omega$, then $\operatorname{Sign}(\Omega)=-\operatorname{Sign}(q)=\operatorname{Sign}(\psi)$ and $\operatorname{Sign}(\psi \Omega)>0$.

The relations (9)-(12), (16) as well as (17), (18) jointly point to reality of correlations $T_{e x c}{ }^{*}>T$ on $0<e \psi \Omega<\varepsilon_{j}$, as well as $T_{e x c}{ }^{*}<<T$ on $e \psi \Omega>\varepsilon_{j}$. The concrete corresponding to (17) dependence $T_{e x c}{ }^{*}=f(e \psi \Omega),\left(0<e \psi \Omega \leq 2 \varepsilon_{j}, \varepsilon_{j}=14 e V, T=300 \mathrm{~K}\right)$ is presented with Figure 1.

Let us draw our attention to the fact that according to (17) and regard to being expected character of $e \psi \Omega=f(t)$ during process of relaxation caused by introduction/leading-out of surplus charge into/from medium composition, for example, the population distribution function of excitation temperatures of medium's chemical bonds and electron energy states can be subjected to discontinuity of the type $(+\infty,-\infty)$ or $(-\infty,+\infty)$. This fact among other things can be interpreted as an indirect indication of possibility of development under these conditions of instability of medium's

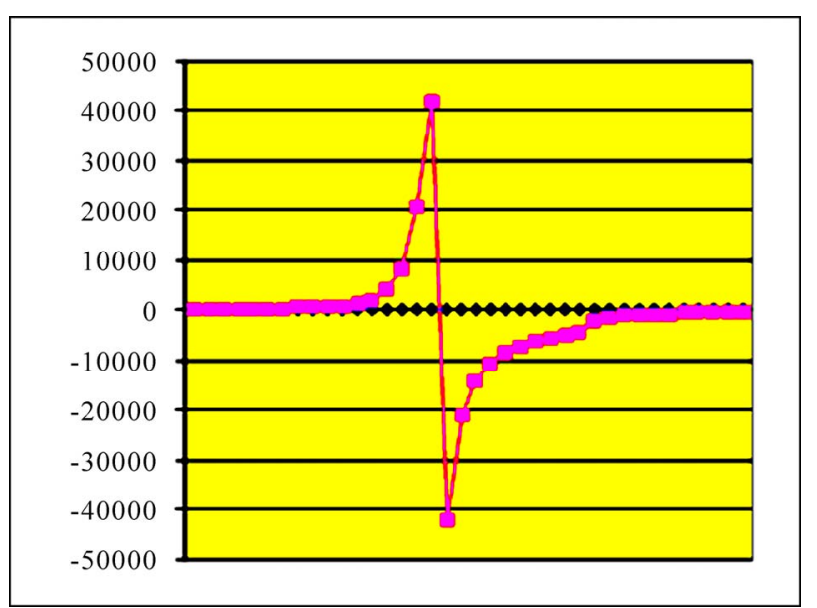

\begin{tabular}{|c|c|c|c|}
\hline$e \psi \Omega$ & $T_{\text {exc }}{ }^{*}$ & $e \psi \Omega$ & $T_{\text {exc }}{ }^{*}$ \\
\hline 0 & 300 & 14,20 & $-21000,0$ \\
\hline 2 & 350 & 14,30 & $-14000,0$ \\
\hline 7 & 600 & 14,40 & $-10500,0$ \\
\hline 9 & 840 & 14,50 & $-8400,00$ \\
\hline 10 & 1050 & 14,70 & $-6000,0$ \\
\hline 12 & 2100 & 16 & -2100 \\
\hline 13,00 & 4200 & 17 & -1400 \\
\hline 13,50 & 8400 & 18 & -1050 \\
\hline 13,8 & 21000 & 19 & -840 \\
\hline $\mathbf{1 3 , 9 *}$ & $42009^{*}$ & 22 & -525 \\
\hline $\mathbf{1 4 , 1 *}$ & $-42000 *$ & 28 & -300 \\
\hline
\end{tabular}

Figure 1. $T_{e x c}^{*}=T \frac{\varepsilon_{j}}{\varepsilon_{j}-e \psi \Omega}=f(e \psi \Omega)_{\bar{s}_{j}}, \varepsilon_{j}=14 e V$. 
atom-molecular structure. The instability, as it might expect, being acceptable to manifest itself, with losses of electrons steadiness on the orbit and even with absorption of the ones by nuclei.

Common peculiarities of IEEC already at this stage testify to real perspectives to form on the ones' base of conceptual views model of BL. Let us forestall however the attempt with both clarification of favourable using conditions of chargeous channel for controlling over material media states AND additional confirmation of validity of just IEEC themselves as base for the working out of supposed model of BL.

\section{Temperature Limitations of IEEC Efficiency}

Verification of temperature diapason that potentially not excluding at least the ensuring possibility of significant changes with chargeous influences of medium's characteristics, all other things being equal, is of special importance for validity to use IEEC as base in forming of true notion of BL.

To make conditions for effective using of revealed "E-channel" more precise, quasi equilibrious plasma's characteristics as applied to concrete parametric nonuniformity in MGD-channel with sectioned electrodes (Figure 2) were estimated versus temperature, all other things being the equal, just as by traditional (generally accepted) method, so by method with account of revealed IEEC.

The values next were compared relative each other. It was taken into account in this case that if a temperature corresponds to diapason where using of ECC is effective one so the differences in levels of being confronted characteristics must be necessary displayed. In doing so it was assumed that the work regime of MGD generator is stationary and realized with constant pressure.

If the Hall's component of electrical field in the MGD channel $E_{h}=\beta(1-\gamma) u B=-E_{q}=--q / \varepsilon_{0} \approx-e\left(n_{i}^{*}-n_{e}^{*}\right) / \varepsilon_{0}$ then $\left(n_{i}^{*}-n_{e}^{*}\right)=-\beta(1-\gamma) u B \varepsilon_{0} / e$.

In view of (1), (2) $\left(n_{i}{ }^{*}-n_{e}{ }^{*}\right)=-\psi n_{i}{ }^{*}=-\alpha^{*} \psi n_{a}{ }^{0}$ whence $n_{a}{ }^{0}=\beta(1-\gamma) u B \varepsilon_{0} /\left(e \alpha^{*} \psi\right)$. But $n_{a}{ }^{0}$ (see (1), (2)) complies also with expression $n_{a}{ }^{0}=P_{\Sigma} /\left\{\left[1+\alpha^{*}(1+\psi)\right] k T\right\}$.

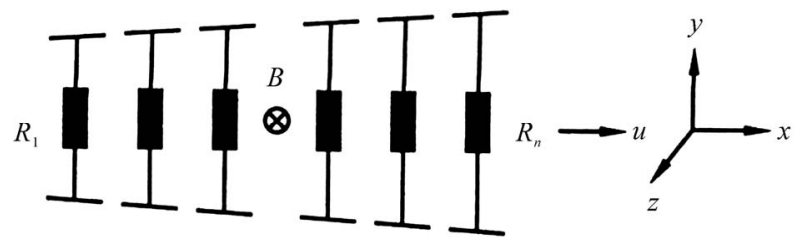

Figure 2. MGD channel.
Then $\frac{1+\alpha^{*}(1+\psi)}{\alpha^{*} \psi}=\frac{e P_{\Sigma}}{\beta(1-\gamma) u B \varepsilon_{0} k T} \quad$ what upon calling $\frac{e P_{\Sigma}}{\beta(1-\gamma) u B \varepsilon_{0} k T}=C$ is transformed into form $\alpha^{*}=1 /[\psi(C-1)-1]$. Potential $\Omega$ was evaluated with $\Omega=E_{h}\left(L_{c h}-x\right)=\beta(1-\gamma) u B\left(L_{c h}-x\right)$ what is in keeping with $e \psi \Omega>0$ (!!!).

The equation system

$$
\begin{aligned}
& \frac{\alpha^{*(2+\psi)}(1+\psi)^{(1+\psi)}}{\left(1-\alpha^{*}\right)\left[1+\alpha^{*}(1+\psi)\right]^{(1+\psi)}}=\frac{1}{P_{\Sigma}^{(1+\psi)}} \frac{g_{i}}{g_{a}} \times \\
& \times\left[g_{e} \frac{\left(2 \pi m_{e}\right)^{3 / 2}(k T)^{5 / 2}}{h^{3}}\right]^{(1+\psi)} \exp \left(-e \frac{V-\psi \Omega}{k T}\right) ;
\end{aligned}
$$

$C=\frac{e P_{\Sigma}}{E_{x} \varepsilon_{0} k T} ; \quad \alpha^{*}=\frac{1}{\psi(C-1)-1} ;$

$\Omega=\beta(1-\gamma) u B\left(L_{c h}-x\right)$

under certain values of $P_{\Sigma}, T, L_{c h}, x$, medium composition and regime of MGD generator is closed.

The system was used to estimate both the levels and dependences character with temperature of ionization parameters of medium that, in accordance with IEEC, correspond to quasi-equilibrium conditions in MGD channel. Related parameters of media in quasi neutral states, all other things being same, were estimated too.

The presented below with Figure 3 results (along with obtained as a whole) were being analyzed under assumption that using of chargeous influences for controlling of material media states will be the most effective in the temperature ranges where, all other factors being the same, just essential differences between estimations of quasi equilibrium medium's ionization parameters are revealed depending on is accounted or no uncompensated electric charge in the medium composition.

The results of Figure 3 correspond to conditions: pressure $P_{\Sigma}=10^{5} \mathrm{~N} / \mathrm{m}^{2}$, flow velocity $u=10^{3} \mathrm{~m} / \mathrm{s}$, Hall parameter $\beta=2$, load parameter $\gamma=0$. Being conditionally taken into account here media correspond to $\mathrm{K}, \mathrm{Li}, \mathrm{H}, \mathrm{Ar}(a, b, c, d$ correspondingly).

During investigations as a whole the following parameters were being modified over ranges: a conventional medium composition $V \in(3.89,24.58) W / A$; pressure $P_{\Sigma} \in\left(10^{5}, 10^{6}\right) \mathrm{N} / \mathrm{m}^{2} \quad ; \quad$ flow velocity $u \in\left(10^{3} .2 \cdot 10^{3}\right) \mathrm{m} / \mathrm{s}$; Hall's parameter $\beta=1,2,4$; load parameter of MGD generator $\gamma=0,0.5$.

The taken into account range of temperature changes was $T \in(300,3000) K$. It was assumed that magnetic induction $B=1.6 T$, channel length $L_{c h}=1 \mathrm{~m}, x=0 \mathrm{~m}$.

It was determined in this connection that significant differences between of being so compared ionization 


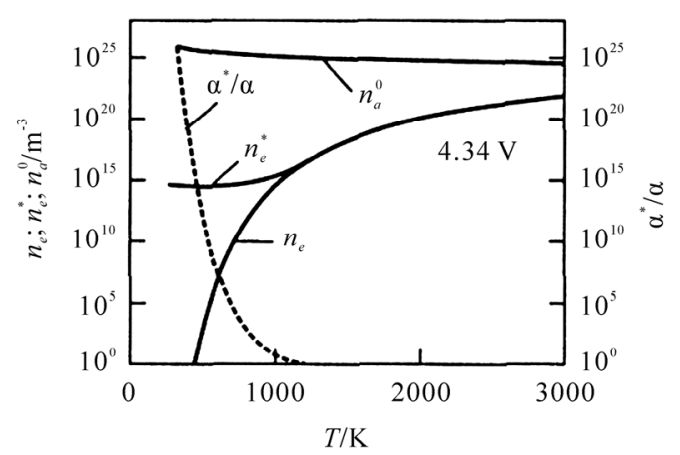

(a)

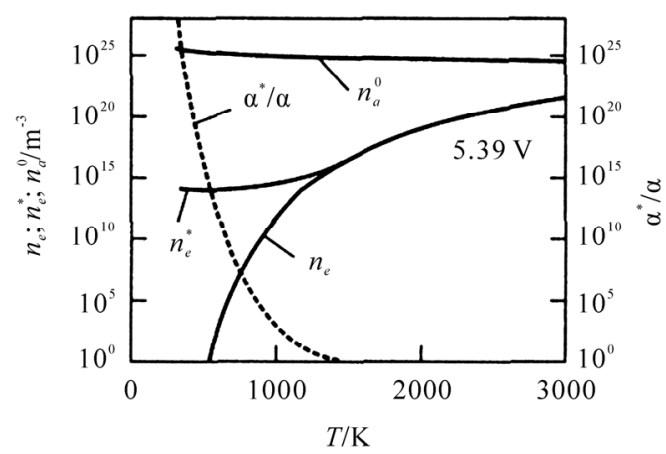

(b)

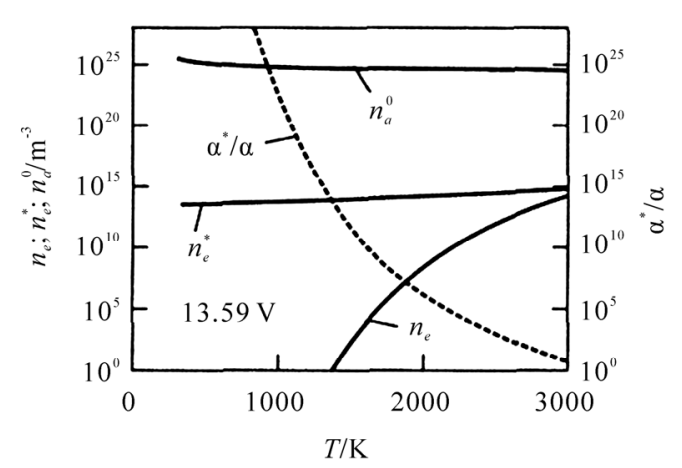

(c)

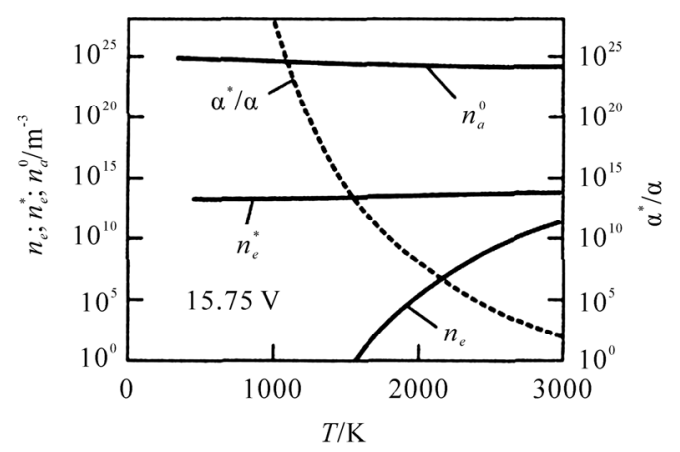

(d)

Figure 3. Characteristic comparison results (conditionallyK, LI, H, Ar). parameters are principally revealed only in field of relatively low temperatures.

It is elucidated that differences of medium composition towards increase of ionization potential make to extension of favorable temperature diapason where using of chargeous influences might be effective for controlling of material media's states.

By confrontational analysis it was revealed that, even with account of wide variation of media compositions, temperature diapason, where an essential differences of the results are disclosed, is limited from the top and also envelopes by itself only oblast of relatively low temperatures.

Even after such seemingly optimistic generalization, let draw our attention and up to basis of the work-IEEC that was only recently pioneered by authors, till now received no of sufficiency diffusion and in itself, in one or other degree, is in need of some additional confirmation.

The results of Figure 3 correspond to conditions: pressure $P_{\Sigma}=10^{5} \mathrm{~N} / \mathrm{m}^{2}$, flow velocity $u=10^{3} \mathrm{~m} / \mathrm{s}$, Hall parameter $\beta=2$, load parameter $\gamma=0$. Being conditionally taken into account here media correspond to $\mathrm{K}$, Li, H, Ar ( $a, b, c, d$ correspondingly).

During investigations as a whole the following parameters were being modified over ranges: a conventional medium composition $V \in(3.89,24.58) \mathrm{W} / \mathrm{A}$; pressure $P_{\Sigma} \in\left(10^{5}, 10^{6}\right) \mathrm{N} / \mathrm{m}^{2}$; flow velocity

$u \in\left(10^{3} .2 \cdot 10^{3}\right) \mathrm{m} / \mathrm{s}$; Hall's parameter $\beta=1,2,4$; load parameter of MGD generator $\gamma=0,0.5$.

The taken into account range of temperature changes was $T \in(300,3000) K$. It was assumed that magnetic induction $B=1.6 T$, channel length $L_{c h}=1 \mathrm{~m}, x=0 \mathrm{~m}$.

It was determined in this connection that significant differences between of being so compared ionization parameters are principally revealed only in field of relatively low temperatures.

It is elucidated that differences of medium composition towards increase of ionization potential make to extension of favorable temperature diapason where using of chargeous influences might be effective for controlling of material media's states.

By confrontational analysis it was revealed that, even with account of wide variation of media compositions, temperature diapason, where an essential differences of the results are disclosed, is limited from the top and also envelopes by itself only oblast of relatively low temperatures.

Even after such seemingly optimistic generalization, let draw our attention and up to basis of the work-IEEC that was only recently pioneered by authors, till now received no of sufficiency diffusion and in itself, in one or other degree, is in need of some additional confirmation. 


\section{IEEC as Object of Investigation}

\subsection{Common Position}

It was accounted that both character and meaningfulness of waited manifestations of relaxation process being inevitably excited in medium composition at the forming stage of AQN-formation and at the degradation stage of the once alike are jointly conditioned by both up level of $e \psi \Omega$ complex and changes character of the one in the time of relaxation.

It should be waited in this connection that if $T_{e x c}{ }^{*}=f(e \psi \Omega)$ function during the relaxation to (on the stage of AQN-formation forming) or even against IEEC (on the stage of one's disintegration) undergoes discontinuity (that is under conditions when instability of medium's atom-molecular structure becomes possible are see Figure 1) then relaxation manifestation character may be extremely complicated.

It may be suggested in particular that at this case regardless of the composition, inevitably excited relaxation to/against IEEC may be jointly accompanied by violations of chemical bonds of medium as well as flow out of electrons from upper electron states (steadiness losses of electrons on orbits, ones' downfalls and even absorptions by nuclei with origin of neutrons-analogue of phenomena $K-$ or $e$-cupture), that is by very complicated manifestations jointly not precluding in that number from reactions excitation that produce energy of intra nucleus origin. To substantiate the last (and, hence, IEEC in a whole) the experimental researches results jointly with materials of [9-13] in that number have been used.

\subsection{Investigation Method and Installation}

It was drawn attention to the fact, that according to

$$
\begin{aligned}
q_{\text {sur }}= & \int_{0}^{t} d q=e \cdot f_{a} \int_{0}^{t}\left[\Phi_{i}^{a \rightarrow m}+\Phi_{e}^{m \rightarrow a}\right] d t- \\
& -e \cdot f_{c} \int_{0}^{t}\left[\Phi_{e}^{c \rightarrow m}+\Phi_{i}^{m \rightarrow c}\right] d t \neq 0
\end{aligned}
$$

surplus charge in volume of some reactor (Figure 4) could have be done, for example, by impulse of capacitor discharge owing to differences between quantity of electricity being brought in reactor during time " $\mathrm{t}$ " (through part of chain "positively charge cover of capacitor-electrode-anode in rector") and, opposite, being brought out from reactor during the same time (through part of chain "electrode-cathode in reactor-negatively charged cover of capacitor).

In (19): $\Phi_{j}=f(t),(j=e, i)$-specific flow of carriers of charge (electrons, ions) on boundaries of media division parts of electrical chain $a \leftrightarrow m, \quad c \leftrightarrow m$ (anode $\Leftrightarrow$ medium, cathode $\Leftrightarrow$ medium respectively).

By Figure 5 block-schema of experimental installation is shown.

In the one's composition: reactor 1 fitted out with electrodes "A", "C" (basic ones), picking out reaction zone and being used for formation of controlling influences onto medium in reactor and, sometimes (what isn't necessarily), with "SE" (subsidiary one) for any information leading-out from reactor; system 2 of medium storage and preparation of one's wanted composition; systems 3 of injection/ejection, additional preparation, ensuring and regulation of barometric characteristics of medium in reactor; power supply system 4; system 5 of registration and measurements of optical, electromagnetic and neutron radiation.

As reactor a vessel done from quartz glass was used (see Figure 6).

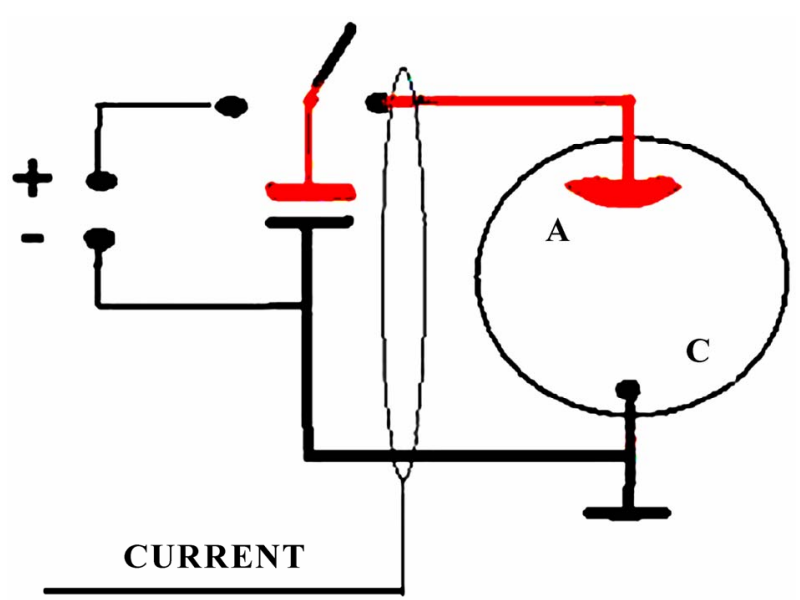

(for registration)

Figure 4. Schema of set for forming and registration of surplus charge.

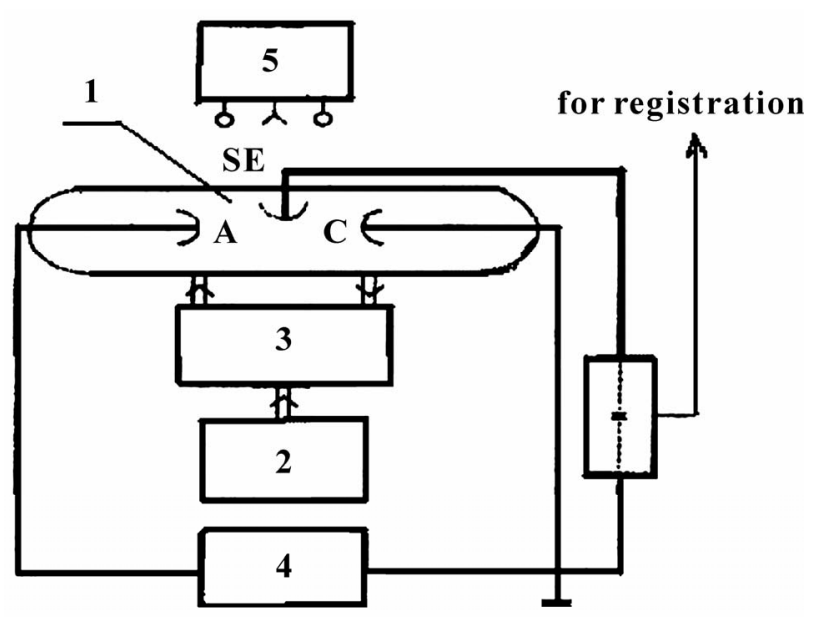

Figure 5. Block-schema of experimental installation. 

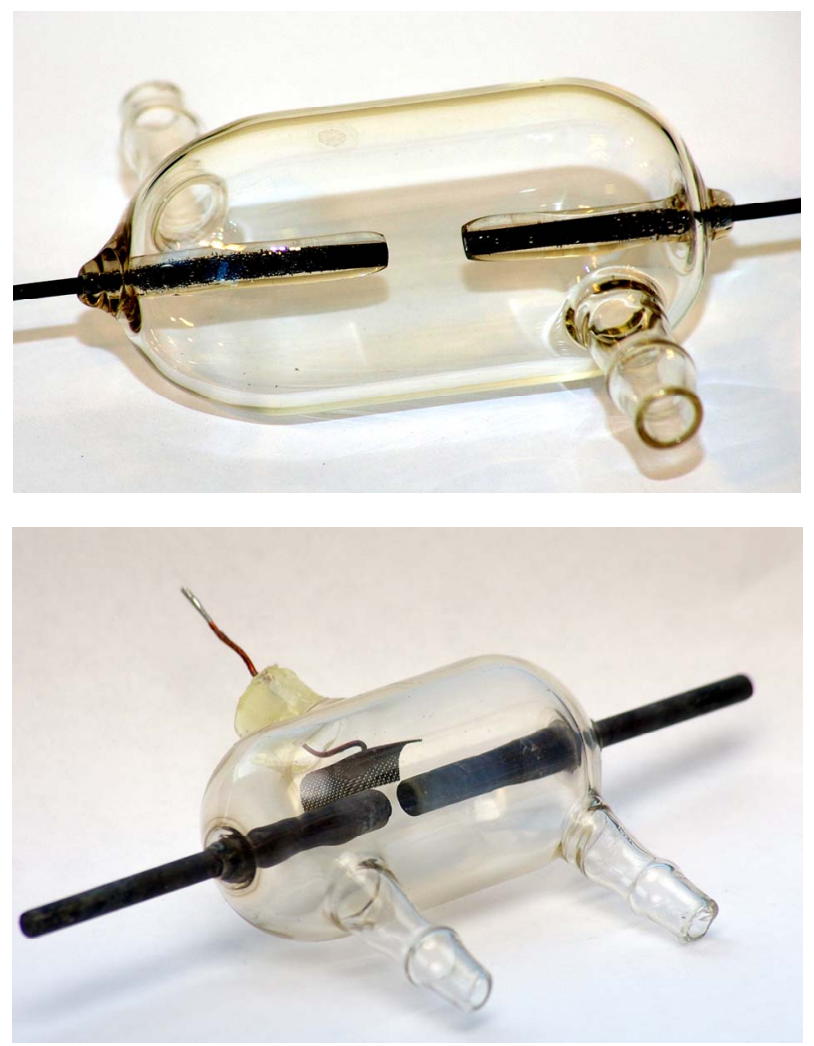

Figure 6. Some reactor variants.

On examinations the reactor was placed in capsule from non-rusting stale (Figure 7) with hollow walls of the one the reactor compartment was picked out.

For providing of controlling influences to medium with energy $2.0-2.5 \mathrm{~m} F$ capacitor was used. The potential differences on the capacitor covers in diapason of $0,8-2,5 \mathrm{kV}$ was varied. Measuring of neutron flow on examinations was foreseen. As measure of neutron flow power on examinations a number of events fixed per discharge impulse was accounted.

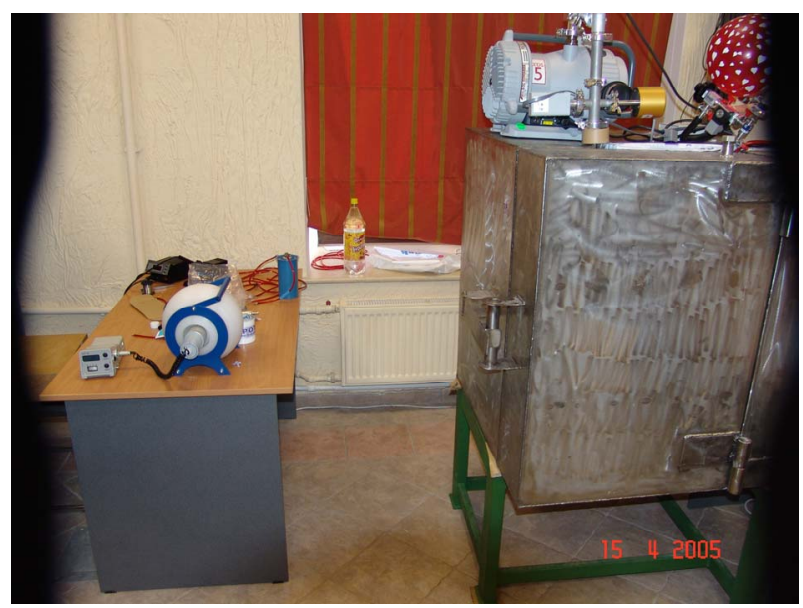

Figure 7. Some sets of installation.

\subsection{Common Results}

Figure 8 illustrates typical experimental oscillograms of tension and current between basic reactor electrodes on examinations. Current-below curve, $227 \mathrm{~A} / \mathrm{div}$, tensionupper curve, $500 \mathrm{~V} /$ div.

As applied to concrete conditions of receiving of curves Figure 8, both the electrical field laying onto discharge interval of the reactor and switching on of oscillograph were carried at the same time with help of special electron key being brought in feeding chain of discharge zone of reactor.

As material of electrodes Mo, W and/ or Kovar (Fe, Ni, $\mathrm{Co})^{3}$ were used. Any dependence of results from electrodes material was not revealed.

As applied to concrete conditions of the oscillograms receiving the application of electrical field to the anodecathode gap and triggering of the oscillograph were being at the same time with the help of electronic key, being introduced into the feeding chain of the reactor.

The initial level of voltages on the capacitor's plates was supported in the frame of being took out diapason and practically was not varied beyond the medium composition from number of being used ones (hydrogen, helium, nitrogen, air, nitrogen-hydrogen mixture with molar relation $\sim 1: 1$ ).

Under conditions being typical ones for Figure 8, from 100 to 200 events per discharge impulse were fixed at distance 0,5 meter from reactor (in general neutron radiation was able to be fixed up to 10 meter from reactor). The trustworthiness of conclusion that being Registered radiation is really neutron one has been confirmed with additional investigations [9,11-13].

For verification of radiation nature and exception so of possibilities to distort results, in connection with influence to detector of one or other strays pick-up, the experimental tests have been done. So the additional analogous block but with distant scintillating target was taken up position side by side with detection block. This additional block detected no events during discharge. Moreover, the hollow walls of operating compartment with hollow's thickness $\sim 250 \mathrm{~mm}$ were filled up with saturated water-solution of boron acid and were used as a special absorbent of neutrons. An introduction of this absorber between reactor and detector was accompanied by sharp weakening (by one order) of having been before registered, all other being equal, neutron flow. In carry- ing out of control experiments, when the hollow intra- walls was empty or even filled up with distilled water, a weakening of neutron flow was not observed. We can state so with full assurance that fixed radiation is really neutron one.

\footnotetext{
${ }^{3}$ Change of material at that case was determined in essence only with technological limitations, connecting with covering of side surfaces of electrodes by glass.
} 

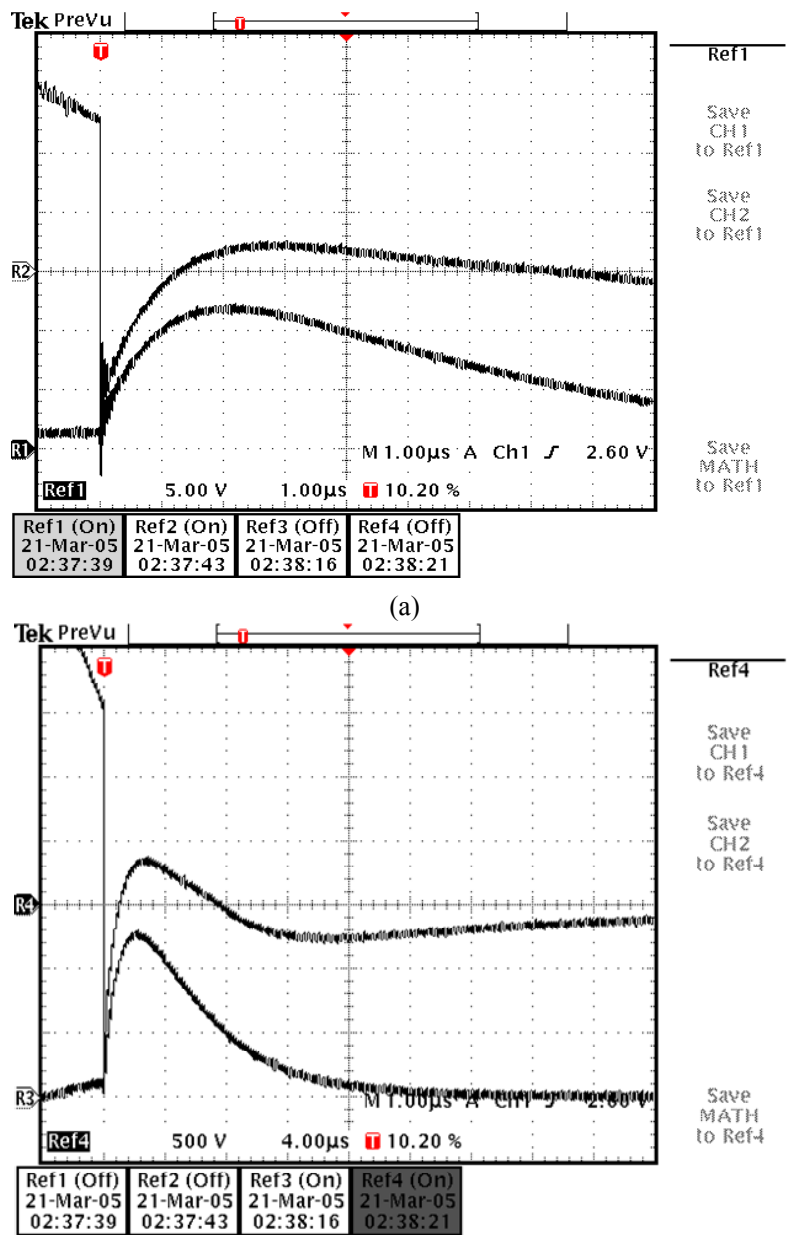

(b)

Figure 8. Typical discharge on pressure: 0.5-1.5 Torr $\left(\mathrm{H}_{2}\right.$, He), 1.0-2.0 Torr $\left(\mathrm{N}_{2}\right.$, air, $\mathrm{N}_{2}+\mathrm{H}_{2}$, molar ratio $\left.\sim 1 / 1\right)$. Scanning: (a) $1 \mathrm{~ms} / \mathrm{div}$; (b) $4 \mathrm{~ms} / \mathrm{div}$.

Let us note also that registration of neutron radiation safely testifies to exciting under these conditions of energy producing reactions of intra nuclear origin too.

The pressure diapason corresponding to fixed neutron radiation is set with experiment. To substantiate importance of arising so in reality of search stage in common cycle of the work let us note that under the pressure above 20 Torr, all other things being equal, discharge of the capacitor takes of the classical shape (Figure 9). Neutron radiation under such conditions is not found.

Let us draw an attention that by oscillograms of Figure 8 the overloading of capacitor's covers with electrical charge of opposite sign is fixed. The last testify to that owing to chargeous influences to medium in that case an untraditional source of energy in the reactor is formed. The last shows so to principal possibilities to accompany the chargeous influences onto medium with leading-out of energy from reactor. Notice that presented with Figure $\mathbf{1 0}$ data testify to such conclusion.

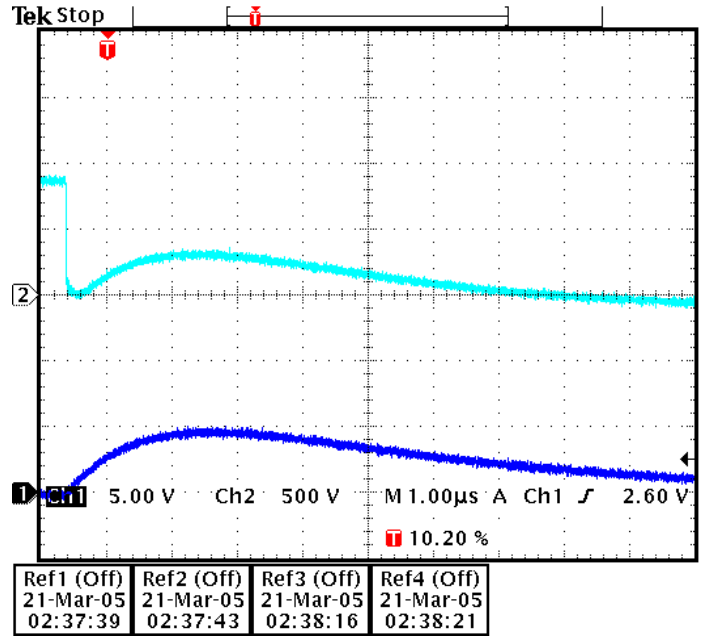

Figure 9. Discharge characteristics under $P=25$ Torr (1-current; 2-tension on capacitor covers).

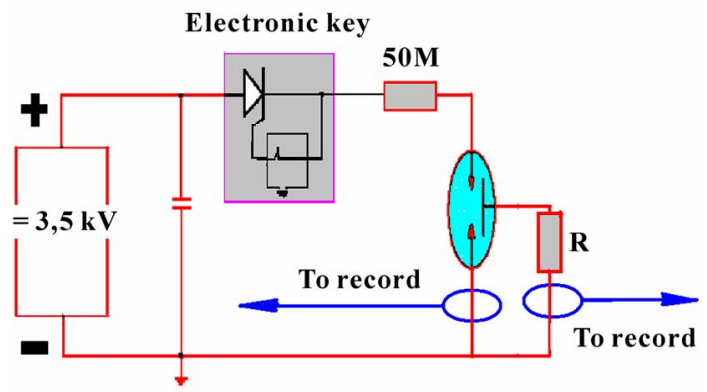

(a)

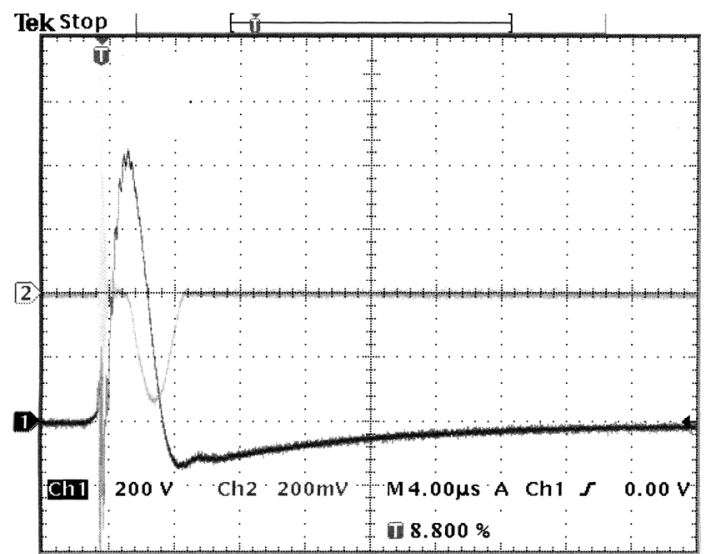

(b)

Figure 10. The installation schema (a) and experimental data; (b) 1-tension: 2-current on/from E-electrode, 0.022 V/A.

The received so experimental results is typical to all investigations totality and, as it was assumed, in the frame of used media nomenclature do not display any once' dependence from medium composition. In other words, common rightfulness of IEEC themselves as well 
as of attempts to form, on the ones' base, model physics views about $\mathrm{BL}$ received so experimental confirmation too. By received results so not only the validity of waited manifestations of chargeous influences to medium but and additionally, on this time just with experimental way, truth and fundamental character of IEEC itself as basis of this work were confirmed too.

Let us further in consecutive order consider key problems of BL.

\section{BL Model Views Forming}

\subsection{Sphericity}

The sphericity substantiation of BL (as a foundation with higher, in respect to the environment, temperature) is traditionally considered as extremely complicated one for solution (if not being defied explanation by ordinary means at all [19]).

In the framework of the developed point of view (i.e.. when it is possible to assume that $T_{b l}$-translation temperature of $\mathrm{BL}$ and $T_{e n v}$-a temperature of environment are close to each other) the existence possibilities verifycation of material AQN-formations (after referred to as BL-formations or BLs) localized in the space is taking however some new tinge.

With the availability in medium of dipole molecules with moment $d$ and at a concentration $n_{d}=\chi n_{\Sigma}=$ $\chi P_{\Sigma} /\left(k T_{b l}\right)$ ( $\chi$-the relative dipoles concentration) the surface tension forces energy will be determined by value

$$
Q_{s t}=U(r) r S_{s f} n_{d}=4 \pi R_{b l}^{2} \frac{d^{2}}{\varepsilon_{0}}\left(\chi n_{\Sigma}\right)^{5 / 3}
$$

If needed expenditures to retain the electric charge in BL composition to be estimated by means of energy accumulated by the BL sphere being under consideration as spherical condenser $\left(T_{b l} \approx T_{\text {env }}\right)$

$$
Q_{c}=\frac{q^{2}}{C}=\frac{q^{2}}{8 \pi \varepsilon_{0} R_{b l}}
$$

then the greatest surplus charge being kept in the sphere of BL may be determined with

$$
\begin{aligned}
& q=4 \sqrt{2} Z_{j} \pi d R_{b l}^{3 / 2}\left(\chi n_{\Sigma}\right)^{5 / 6}, \\
& Z_{j}=\left\{\begin{array}{c}
1, \text { if } j=i ; \\
-1, \text { if } j=e .
\end{array}\right.
\end{aligned}
$$

The excess electron concentration in BL medium complying with $q$

$$
\Delta n_{q}=-\frac{q}{e V_{b l}}=-\frac{3 \sqrt{2} Z_{j} d\left(\chi n_{\Sigma}\right)^{5 / 6}}{e R_{b l}^{3 / 2}} .
$$

Both physical meaning and level of potential $\Omega$ would be determined in that case by the greatest negative work has been made by BL-medium itself when carrying in of the surplus charge into BL sphere. So if the work has been done with $\mathrm{BL}$ medium is $L=-q^{2} / C=q \Omega$ then electrical potential will be determined as

$$
\Omega=-q / C=-\frac{\sqrt{2} Z_{j} d R_{b l}^{1 / 2}\left(\chi n_{\Sigma}\right)^{5 / 6}}{2 \varepsilon_{0}} .
$$

For a case when the sphere radius of BL-formation $R_{b l}=0,075 \mathrm{~m}$ the absolute level of charge $q$ held in BL-medium (a), electric potential $\Omega$ (b) and energy accumulated by the BL sphere as a capacitor (c) versus relative dipoles keeping are shown at the Figure 11.

The analogous characteristics, but versus BL's sphere radius and for case when relative dipoles keeping $\chi=0,01$, are presented at the Figure 12 .

Thus the possibilities for energy ensuring of BLs' sphericity even in a case when relative dipoles keeping in a medium corresponds to relatively low are confirmed by presented results.

\subsection{Energy Problem}

\subsubsection{Common Remark}

$\mathrm{BL}$ origin in form of material formations with uncompensated electric charge is made possible by only in connection with manifestations of external disturbances with one or another nature onto medium (the ones not considered by us within this work). Similarly, the existence proper of BL itself likewise is realized under BLenvironment interactions.

In other words, the ball lightning presents by itself, first of all, thermodynamically opened system the properties of the one are typical for significantly upset medium formations and are necessary supported by especial processes aggregate (complex) of heat and mass transfer as between energy states of different physical nature of BL medium, so between BL as a whole and environment.

Accounting significance of the noted let us underlines once again that, in framework of the work, the revealed Conditions of Inner Energy Equilibrium are wise to regard, before all, not as the postulate directly indicating the concrete characteristics of AQN formations but, to a greater extent, only as the physical appropriateness complex, justifying the excitation necessity of relaxation in AQN-medium composition towards new conditions of inner energy equilibrium (having been changed owing to carrying in medium composition of surplus charge) during of very that, with account of the concrete peculiarities of both relaxation proper and interactions between AQN-formations and environment, quasi-stationary characteristics of the AQN-formations themselves are being formed. 


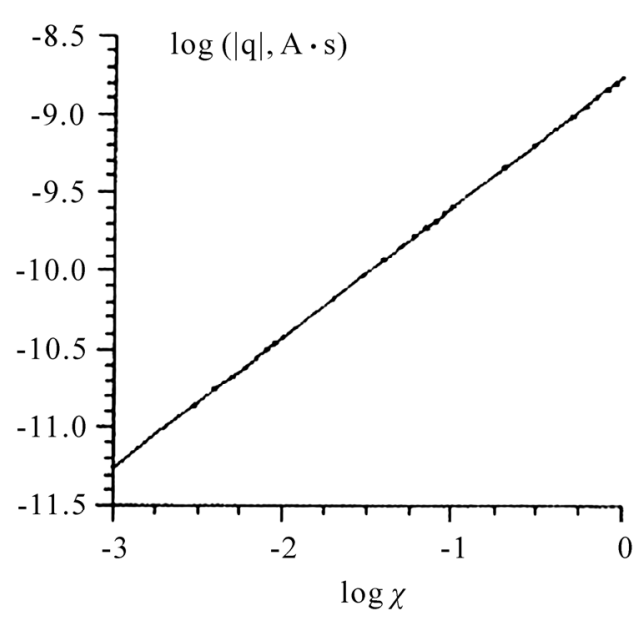

(a)

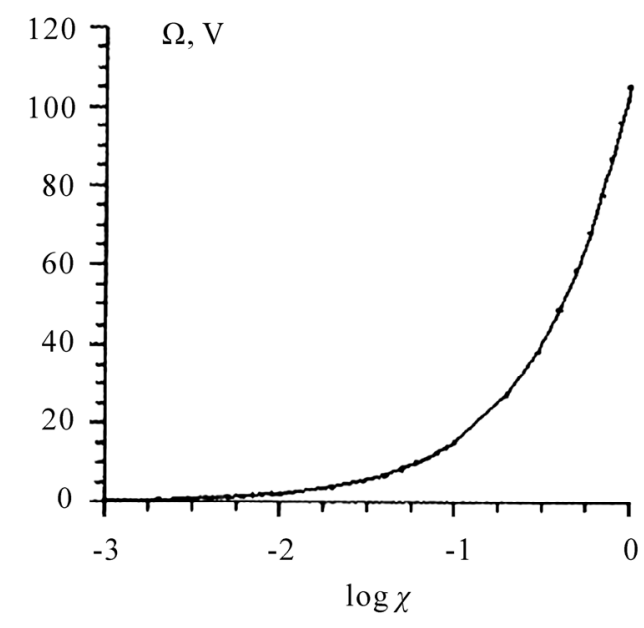

(b)

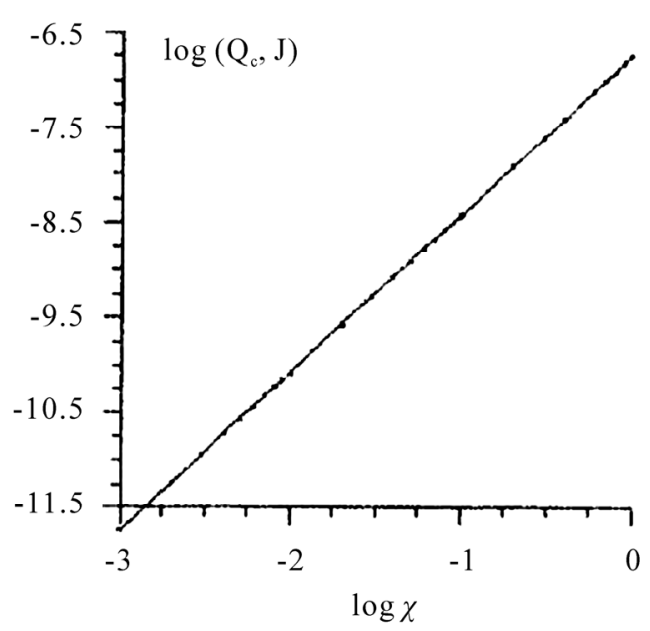

(c)

Figure 11. The holdable charge's characteristics $\log (|q|)$, $\Omega, \log \left(Q_{c}\right)=f(\lambda)$.

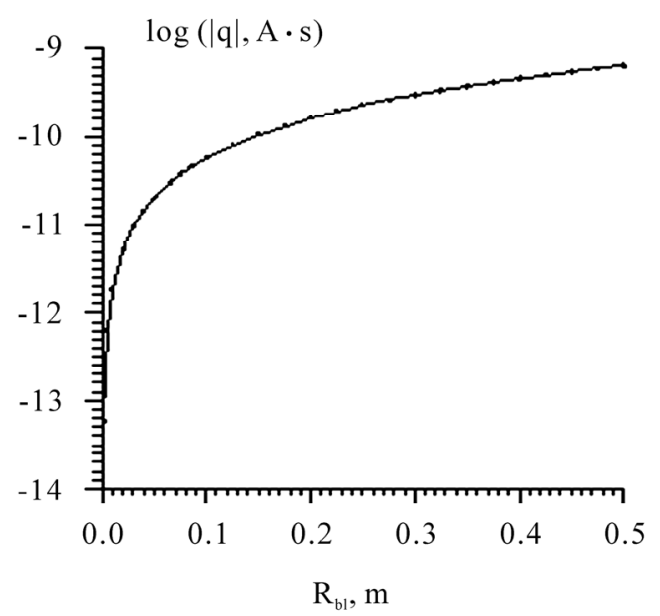

(a)

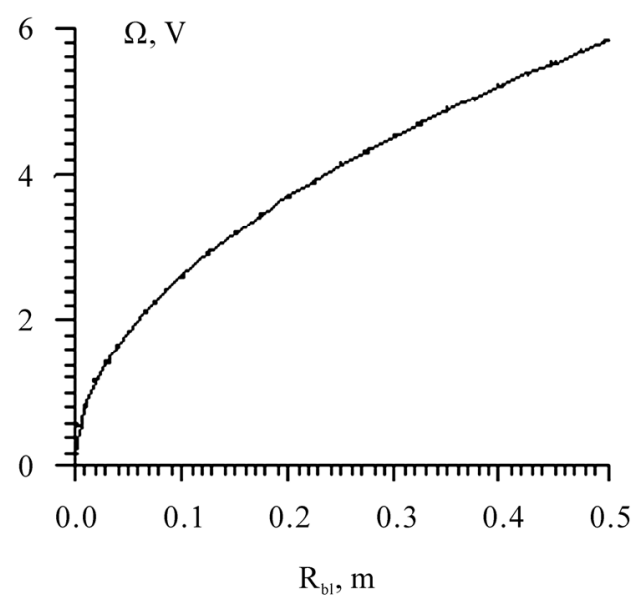

(b)

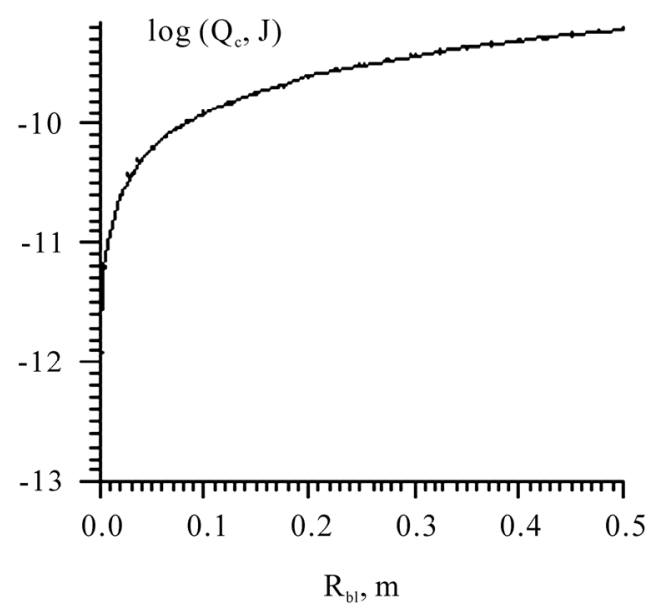

(c)

Figure 12. The holdable charge's characteristics $\log (|\mathbf{q}|), \Omega$, $\log \left(Q_{c}\right)=f\left(R_{b l}\right)$ 


\subsubsection{Radiate Ability Problem}

Within the formed ideas of BL as of material formation with uncompensated electric charge the radiation capacity is substantiated first of all by typical for aquasineutral formations of excesses of chemical bonds and electron energy states excitation temperatures $\left(T_{\text {exc }}{ }^{*}\right)$ over translation one $\left(T_{b l}\right)$ (in that number and especially on the distant approaches to the equilibrium conditions, see (6.2.1) and (17), (18))

In support of practical significance of the waited reflections in reference to the conditions that are similar to Figures 11, 12 a quantitative estimations of quasi-equilibrium parameters of different composition media has been received.

The estimation was being conducted on the base of system including both ionization parameters bonds equations of plasma medium within of three liquid model [17] and expressions having been received in the work. Specifically the system was included following equilibriums:

$$
\begin{aligned}
& n_{a}{ }^{(0)}=P_{\Sigma} /\left\{\left[1+\alpha^{*}(1+\psi)\right] k T_{b l}\right\} ; \quad n_{\Sigma}=P_{\Sigma} /\left(k T_{b l}\right) ; \\
& q=4 \sqrt{2} Z_{j} \pi d R_{b l}{ }^{3 / 2}\left(\chi n_{\Sigma}\right)^{5 / 6} ; n_{d}=\chi n_{\Sigma} ; \quad n_{i}{ }^{*}=\alpha^{*} n_{a}{ }^{(0)} ; \\
& \Delta n_{q}=-\frac{3 \sqrt{2} Z_{j} d\left(n_{d}\right)^{5 / 6}}{e R_{b l}^{3 / 2}} ; \quad \psi=\Delta n_{q} / n_{i}^{*} ; \\
& \Omega=-\frac{\sqrt{2} Z_{j} d R_{b l}{ }^{1 / 2}\left(n_{d}\right)^{5 / 6}}{2 \varepsilon_{0}} ; \quad T_{e x c}{ }^{*}=T \frac{\varepsilon_{j}}{\varepsilon_{j}-e \psi \Omega} \\
& \frac{\alpha^{*(2+\psi)}(1+\psi)^{(1+\psi)}}{\left(1-\alpha^{*}\right)\left[1+\alpha^{*}(1+\psi)\right]^{(1+\psi)}}=\frac{1}{P_{\Sigma}^{(1+\psi)}} \frac{g_{i}}{g_{a}} \times \\
& \times\left[g_{e} \frac{\left(2 \pi m_{e}\right)^{3 / 2}(k T)^{5 / 2}}{h^{3}}\right]^{(1+\psi)} \exp \left(-e \frac{V-\psi \Omega}{k T}\right) ;
\end{aligned}
$$

Among unknowns in solution of the system ten following parameters are accounted: $n_{a}^{(0)}, n_{\Sigma}, \alpha^{*}, \psi, n_{d}, \Delta n_{q}, n_{i}^{*}, q, \Omega$, $T_{e x c}{ }^{*}$. The parameters $\chi, R_{b l}, V, Z_{j}$ were being varied. It was assumed that $P_{\Sigma}=1.01325_{10^{5}} P a, T=300 \mathrm{~K}, d=1 \mathrm{D}$. The system so was being closed.

The revealed so ionization degrees $\alpha^{*}$ of different composition media (a) in common with the both excite temperatures of electron energy states $T_{\text {erc }}$ " (b) and levels corrections of ionization potentials being stipulated by presence in media composition of surplus charge $\psi \Omega$ (c) delivering the ionization degree versus relative dipole keeping in media composition of BL with $R_{b l}=0.075 \mathrm{~m}$ are presented at Figure 13.

The curves 1-6 conform to conventional media composition with $V=6,8,10,12,14,16 \mathrm{~W} / \mathrm{A}$ (curves 1-6 accordingly). Plots of Figure 14 present the dependences

$\overline{{ }^{4} \text { In so doing will bear in mind that, under generally accepted views, }}$ "any negative temperature $T_{\text {exc }}$ " is hotter relative any positive one".

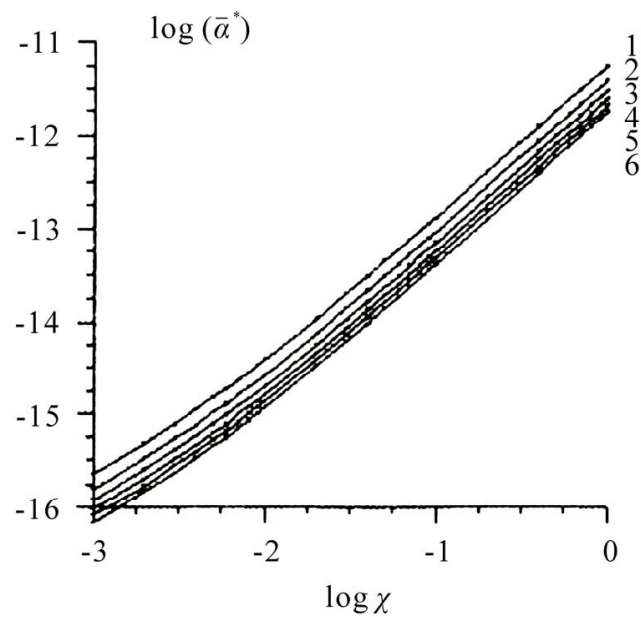

(a)

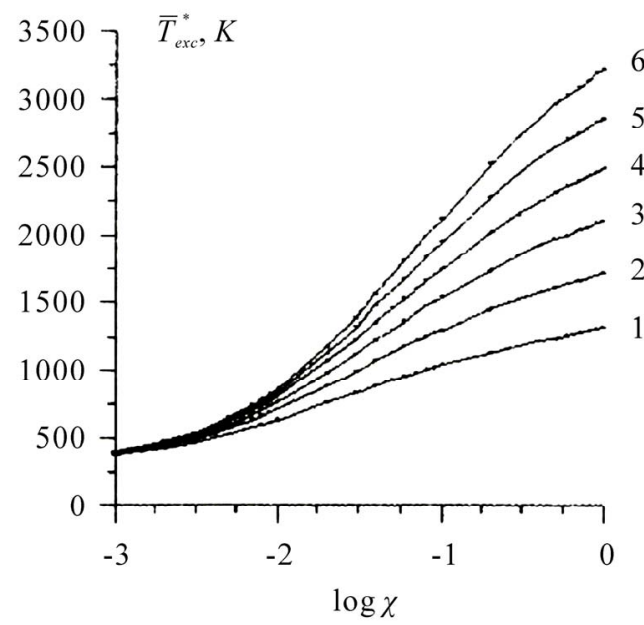

(b)

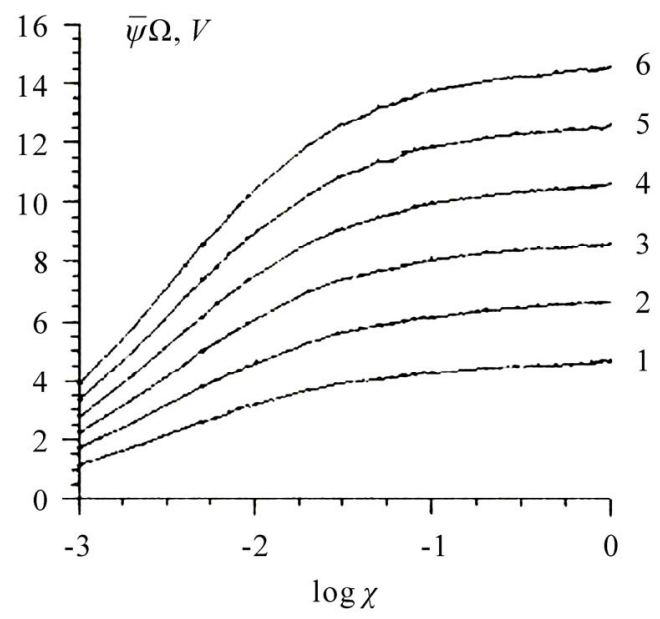

(c)

Figure 13. The quasi-equilibrium characteristics $\log \left(\bar{\alpha}^{*}\right)$, $\overline{\boldsymbol{T}}_{\text {exc }}, \bar{\psi} \Omega=f(\log \chi)$. 


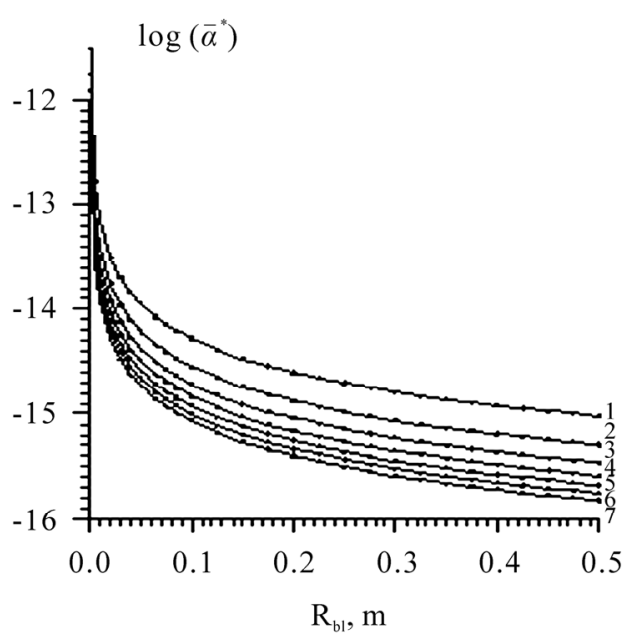

(a)

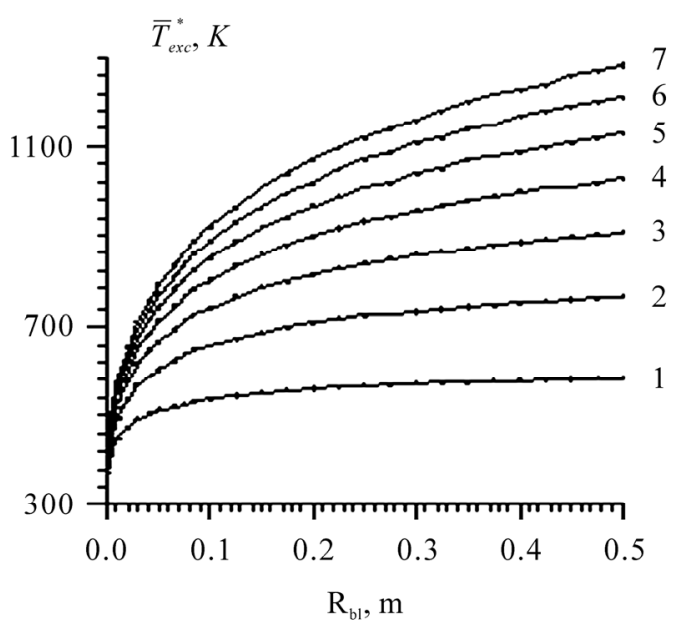

(b)

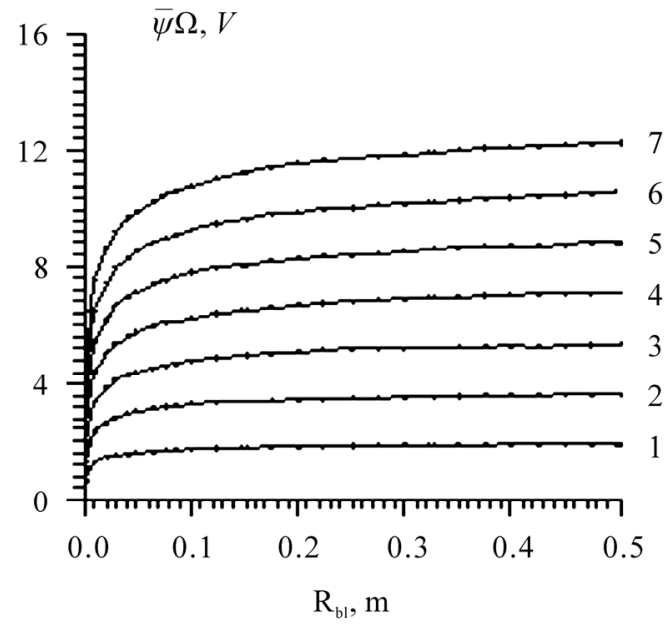

(c)

Figure 14. The quasi-equilibrium characteristics $\log \left(\bar{\alpha}^{*}\right)$, $\overline{\boldsymbol{T}}_{e x c}, \bar{\psi} \Omega=\boldsymbol{f}\left(\boldsymbol{R}_{b l}\right)$ of the same parameters but versus radius of BL foundation sphere.

The plots conform to conventional media composition with $V=4,6,8,10,12,14,16 \mathrm{~W} / \mathrm{A}$ (curves $1-7$ accordingly). Being taken into account the relative dipole keeping in a composition of $\mathrm{BL}$ medium is $1 \%$ $(\chi=0,01)$.

Let us note that, as applied to no equilibrium aquasineutral foundations state, reflecting best of specific character of ones' relaxation under conditions of energy interchange with environment, the absolute values of present-day parameter $|\psi|>|\bar{\psi}|$ and levels $T_{e x c}{ }^{*}>>\bar{T}^{*}$.

In other words, the obtained upper results indicate convincingly as a whole that the natural air foundations with uncompensated charge keeping in ones' composition (BL) not only can exist themselves but also are really able to radiate energy even under conditions, when translation temperature of the foundations is correlated with (or even less than) the environment temperature $\left(T_{b l} \leq T_{e n v}\right)$.

Let us bring into consideration an effective radiation temperature $T_{\text {rad }}$ as parameter determining, on a basis of the generally accepted propositions, radiating power of BL into environment

$$
Q_{\text {rad }}=S_{b l} q_{\text {rad }}=4 \pi R_{b l}{ }^{2} \varepsilon_{g} C_{0}\left(T_{\text {rad }} / 100\right)^{4}
$$

To concretize conditions for numerous estimations of $Q_{\text {rad }}$ will be assumed that $T_{\text {rad }}$ accordingly (25) at the same time correlates with excitation temperature of chemical bonds and electron energy states of some characteristic for BL (within of the ideas about common features along with a typical reactions relaxation composition and energy feeding mechanism) energy level $\varepsilon_{j}{ }^{* 5}$, to say

$$
T_{\text {rad }}=T_{b l} \frac{\varepsilon_{j}^{*}}{\varepsilon_{j}^{*}-e \psi \Omega}
$$

In other words, the temperature $T_{\text {rad }}$ will be considered as a parameter determining, in traditional mind, the radiating power of $\mathrm{BL}$ and, at the same time, as a factor being functionally stipulated by peculiarities of excitation temperature distribution of chemical bonds and electron energy states peculiar to BL media.

It is possible so within of taken into account with (26) bonds the degree of darkness $\varepsilon_{g}$ to be estimated by the ionization degree of a media with $e V=\varepsilon_{j}{ }^{*}$ as determining, in that case, the settlement density of energy states with radiation transitions of electrons from one

\footnotetext{
${ }^{5}$ Admittedly, the accounted so character of bonds between $T_{\text {rad }}$ and energy states excitation temperatures is substantiated essentially, within this work, by only the necessity to refinement conditions for following numerous estimations of $Q_{\text {rad }}$ and in the future calls for a more precise definition.
} 
orbit to another in composition of BL media

$$
\varepsilon_{g}=\frac{\Delta n_{q}}{\psi} \bullet \frac{k T_{b l}}{P_{\Sigma}}
$$

Here $\Delta n_{q}$ - surplus concentration of particles-charge carriers.

\subsection{Octopus's Effect}

The being noted by eyewitnesses capability of BL to overcome different barriers from dielectric materials (window-frame for example) by means of penetration through inanes in the body of barrier (hollows, cracks, splits) being essentially lesser, concerning to BL, sizes and then to restore (reconstruct) in essence the ones' initial shape and characteristic is substantiated too. The one is substantiated, first of all, with susceptibleness of surplus charge in composition of BL's medium to influences of external electric fields and hence with one's obvious capability not only to overcome through barrier (wall) from dielectric material along the channels in barrier's body in hollows (cracks, splits) form, but and then, after penetration through channels, to form (to restore) behind barrier in environment a new AQN-formation with initially corresponding to BL characteristics.

\subsection{The Energy Feeding Source}

The way of attack untwisted in this work opens else real prospects to solve the problem of nature substantiating of the energy source for feeding of BL.

Indeed, as it has been readily apparent from observations, the keeping up (continuous reproduction) of quasi-stationary states in process of BLs existence is realized in that number (unless not always) under conditions when the ones radiate energy into environment.

This seemingly that the radiation of energy into environment must be accompanied by reduction of settlement levels of energy states (relative to stationary settlement level of the ones) with radiation transitions of electrons from one orbit to another and so bring towards lowering of radiating capacity of BL. As applied to stationary state of BL the last however is practically kept constant one. In the framework of the formed method of attack the noted comes to be explicable.

Indeed, it is obviously that the settlements lowering of chemical bonds and electron energy states, in connection with radiation all other things being equal, to be necessary accompanied by (stipulates) an additional excitation (overheating) of "mechanical" energy states of BL medium $\left(T_{b l}\right)$ in relation to the being reduced so excitation levels of the "no mechanical" energy states $\left(T_{e x c} *\right)$.

The growth of misbalance in energy distribution over states in connection with radiation is suppressed so by the energy flow from mechanical energy states towards the ones but being no mechanical nature. Let us draw attention to the fact that the flow of energy from mechanical energy states leads to drop of BL's translation temperature $\left(T_{b l}\right)$ even to the levels below environment temperature $\left(T_{e n v}\right)$ and, in turn, stipulates origin of energy flow from environment to BL.

In other words the both existence and nature of source to feed BL with energy is really substantiated. This source feeding with energy of BL is so environment.

By this means in the framework of the formed model a quasi-stationary state of BL is necessary consistent with conditions when $T_{b l}<T_{e n v}$. Just that relation's range between the noted temperatures provides of BL with heat absorption from environment as clearly indemnifies for ball lightning's energy escape by radiation and essentially makes possible existing of BL in nature as itself. The common picture of exchange energy-mass processes in these conditions in full measure corresponds to both Le Chatelier Principle and the common character of material systems reactions onto disturbances.

\subsection{Energy Feeding Mechanisms}

There can be no doubt that the feeding of BL with energy from environment on a stationary existence stage is realized by traditional mechanisms of energy-mass-exchange. Both convective heat input and input of energy inside BL in connection with mass-exchange between ball lightning and environment are naturally enough to account in components number of the energy supplying (energy feeding) mechanism.

\subsection{Energy Resource Components}

Channels as and sources to form energy-resources of BL along with needed conditions for supplying of the one's quasi-stationary characteristics are also substantiated within the formed ideas model of BL as about localized in the space containing uncompensated electric charge material foundation with differences between translational temperature and excitation temperatures of both chemical bonds and electron energy states.

In account with the revealed appropriateness in equilibriums and common conditions to localize material foundatio9ns in the space, the common level of BL's energy resources is advantageous to estimate in that case with discrete blocks. These are radiation flow energy $Q_{\text {rad }}$, energy saved up by sphere of BL as capacitor $Q_{c}$, nergy $Q_{\varepsilon}$ accumulated with $\mathrm{BL}$ media in account with deformation of settlings distribution function of chemical bonds and electron energy states under relaxation of 
BL-media towards equilibrium conditions accounting of availability of uncompensated electric charge in one's compositions.

\subsection{The Multi-Faces Problem}

Making longer discussion in the framework of the being formed BL model let us attention draw to the fact that the energy absorbed by BL from environment at the same time can be used up at least in two directions. Firstly, it is the one for indemnity of energy losses in connection with radiation. For secondly, it is the one for putting down settlings levels diminution of excited chemical bonds and electron energy states (continuous reproducetion of radiation capacity) stipulated by mass-exchange processes between BL and environment.

It can be assumed that distribution character of feeding energy flow between the noted directions determines the total level of ball lightning's energy resources and, in its turn, is determined by common as well as being specifically established, as the existence conditions of BL require, peculiarities of energy feeding mechanism.

If this the case, the relative contribution of each from energy feeding mechanism's components (convective heat exchange or/and mass transfer) must show essential influence over quasi-stationary characteristics of BL. The dependence of BL foundation's energy resources upon of concrete realized, at each single of occasion, energy feeding mechanism can be considered so as the most probable reason, from assumed ones, of being noted with observations so called multi-faces of ball lightning.

By way of the first step for following consideration let us more concrete formulate ideas upon waited, at each separate case in connection with the noted, common peculiarities of quasi-stationary states of balls lightning. For better visualization of these peculiarities, the needed consideration should be carry out with the supposition of limit contribution into the BL's energy feeding mechanism either of one or another its component from the accounted ones (only of convective heat exchange or, on contrary, only of energy input inside BL from environment in mutual mass-exchange connection with).

Even not accounting of cognitive advisability, allowance of the last is substantiated, among other factors, by possible variations of real environment characteristics and consequently of BL's existence conditions.

\subsubsection{Convective Heat Exchange}

Overpowering contribution of convective heat exchange into energy feeding mechanism of balls lightning with stationary characteristics corresponds to conditions, when BL-environment mass-exchange for the most part has been put down. The quasi-stationary states of BL being existed in the conditions must be characterized first of all by constancy with time of both the radiation characteristics and the settlements of chemical bonds and electron energy states. The energy being supplied to BL from environment must be expended in these conditions only to compensate energy loses of ball lightning with radiation (ideal case).

It should be emphasized that BL-media conforming to quasi-stationarity conditions (and being exceptional so with invariability of energy states and depression of mass-exchange) must be characterized in the case by essential deformation of distribution function of chemical bonds and electron energy states towards internal energy equilibrium with account of surplus electric charge in the media composition.

Let us note that specificity of the being established so distributions points to existence (and possibilities of effective displays) of nontraditional, in the framework of generally accepted physical views, energy resources formation channel of BL. Of the channel being substantiated with obvious capacity of media with surplus electric charge to absorb energy of one or other source to supply the deformation of media's energy states settlements and functioning, in principle, at any stage of ball lighting's vital cycle.

It is safe to assume also that the radiate characteristics of BL in quasi-stationary state under energy feeding by convective heat input (and hence when mass-exchange is depressed) likewise are must be stood out with some typical features.

By significant deformation of energy states settlements, the BL's radiation, in this case, must be mainly realized by transitions in the diapason energy states having been drawn (essentially!) to the internal equilibrium conditions accounting presence of surplus charge in media composition

\subsubsection{Mass-Exchange Energy Feeding}

Contrary to the previous case the power supply of BL by mass-exchange mechanisms necessary assumes that the energy flow from environment to feed with energy of quasi-stationary states of ball lightning is being necessary expended at least towards two ways:

- Firstly, to supply radiation;

- To ensure with energy the continuous reproduction of energy states settlements that determine the radiate capacity of BL, secondly.

It is possible to assume also that radiate capacity of BL-medium is substantially (most probably) manifested in the field of distant approaches to equilibrium conditions and is realized, in that case, by radiate transitions between energy states with essentially higher levels of excitation temperatures (the ones at the relaxation stage 
undergo a rupture of $(-\infty,+\infty)$ kind). But the BL's energy resources component conditioned by settlements distribution function deformation of chemical bonds and electron energy states with relaxation most likely in the case is kept only partly filled up.

\subsection{Preliminary Result}

The preliminary examination materials testify that account of revealed [1-13], earlier unknown peculiarities of inner energy equilibrium conditions of media with surplus (uncompensated) electric charge opens truly up new prospects for adequate ideas model substantiation of BL. The last however requires for addition confirmation rather by numerous estimations.

\section{BL's Quasi-Stationary States}

\subsection{Common Positions}

Will be considered that the BL quasi-stationary state is manifested first of all with immutability in time of its radiate characteristics.

Common conceptions about BL in this conditions will be presided in connection with this by equations complex jointly substantiating just as both the existence reality and energy resources of the ball lightning in form of localized in space material foundation with uncompensated electric charge so others characteristic parameters of the BL versus media compositions and common specificity of feeding energy mechanisms of its quasi-stationary state.

With account of existence of differences channels for keeping/radiation by BL from/into environment the energy resource of BL in quasi-stationary state will be estimated with separate components. Will be taken into account specifically:

- radiation flow $Q_{\text {rad }}$;

- energy $Q_{c}$ having been accumulated by sphere of BL as electric capasitor;

- energy $Q_{\varepsilon}$ having been accumulated under settlements distribution function deformation of chemical bonds and electron energy states of BL medium when relaxing the one towards new equilibrium conditions ${ }^{6}$.

\subsection{Convective Energy Input}

When convective heat input to be considered as the

${ }^{6}$ Existence of the energy resources' component $Q_{\varepsilon}$ corresponds to inner energy equilibrium conditions with account of possible presence of excess charge in media composition and in the framework of generally accepted till now views is not substantiated. mechanism feeding of quasi-stationary state of ball lightning with energy that equation determining the waited power input to BL from environment versus temperatures as titled above is

$$
Q_{h i}{ }^{\text {conv }}=S_{b l} q_{\text {conv }}=4 \pi R_{b l}^{2} \alpha_{c o n v}\left(T_{e n v}-T_{b l}\right) .
$$

Here: $\alpha_{c o n v}$-heat transfer coefficient.

By the conditions of one-to-another equilibrium in value of introducing (to $\mathrm{BL}$ from environment) and radiated contrary (to environment from BL) energy flows will be determined in that case the demands to supply power mechanism feeding. In substantiated limitations so (mass-exchange between the ball lightning and environment is depressed) through correlation of (25) and (28) the conditions of BL's quasi-stationary states supplying with energy (of characteristics invariability) will be determined as

$$
\Delta T_{c o n v}{ }^{*}=T_{e n v}-T_{b l}=\frac{\varepsilon_{g} C_{0}}{\alpha_{c o n v}}\left(\frac{T_{r a d}^{c o n v}}{100}\right)^{4}
$$

As applied to a case of convective heat input the numerous estimations of energy resources of balls lightning under quasi-stationary states as well as feasibility of the lasts by themselves were being conducted within equilibrium approximation (see 5.6.1).

As this take place, with account (26) the effective radiate temperatures were taken equal to quasi-equilibrium excitation temperatures $\bar{T}_{e x c}^{*}$ of electron energy states conforming to ionization energy

$$
T_{\text {rad }}^{c o n v}=\bar{T}_{e x c}^{*}=T_{b l} \frac{V}{V-\bar{\psi} \Omega}
$$

As for relative blackness degrees $\varepsilon_{g}$, the ones, all other factors being the same, were set equal to quasiequilibrium ionization degree $\bar{\alpha}^{*}$ (estimations of $\bar{\alpha}^{*}$ along with of $\bar{T}_{e x c}^{*}$ were presented above by Figures 13, 14). The equation

$$
Q_{\varepsilon}^{c o n v}=\frac{4}{3} \pi R_{b l}^{3} \frac{P_{\Sigma}}{k T_{b l}} e \psi \Omega
$$

was being used to estimate the energy resources component $Q_{\varepsilon}^{\text {conv }}$ of ball lightning in that case.

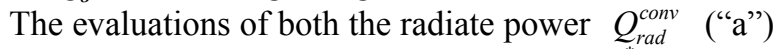
according to (25) and the differences $\Delta T_{\text {conv }}{ }^{*}$ between the ball lightning's translation temperatures and environment temperature ("b") as well as of the BL's energy resources component $Q_{\varepsilon}^{\text {conv }}$ ("c") versus the relative dipoles content in BL's media composition are represent in Figure 15.

In during the estimations it was assumed that BL's sphere radius $R_{b l}=0.075 \mathrm{~m}$. The plots $1-6$ correspond to conventional media composition with $V=6,8,10,12,14,16 \mathrm{~W} / \mathrm{A}$ respectively. As for the rest 


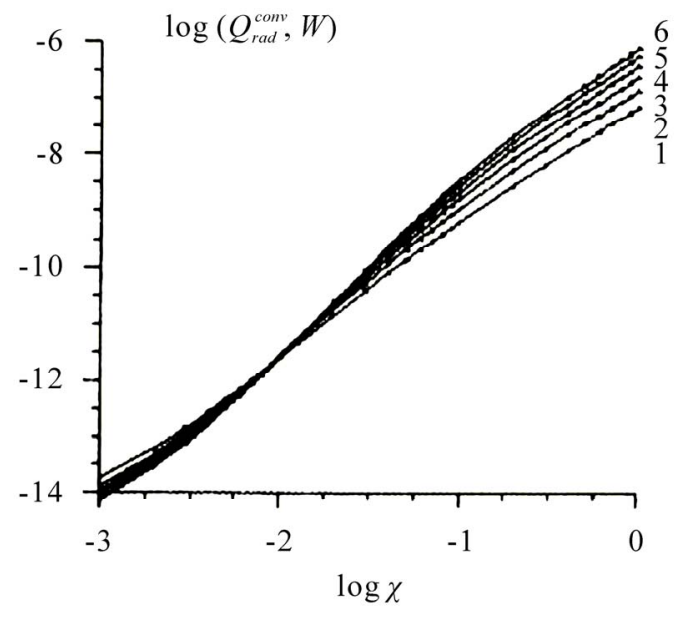

(a)

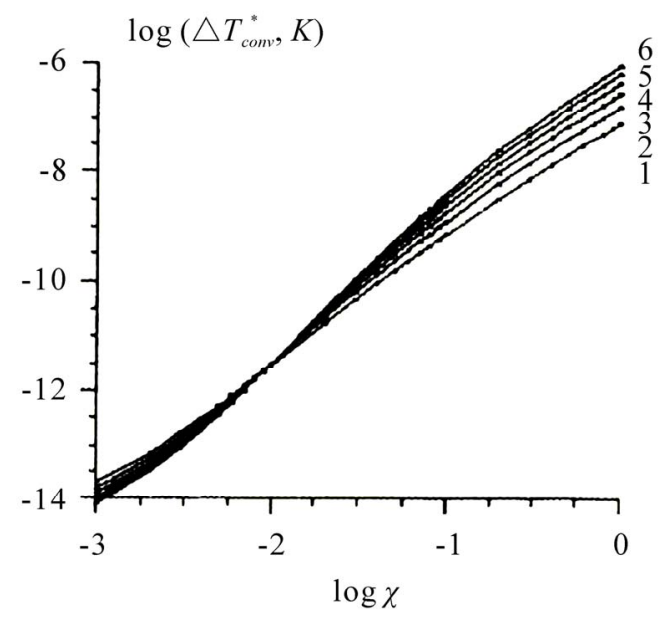

(b)

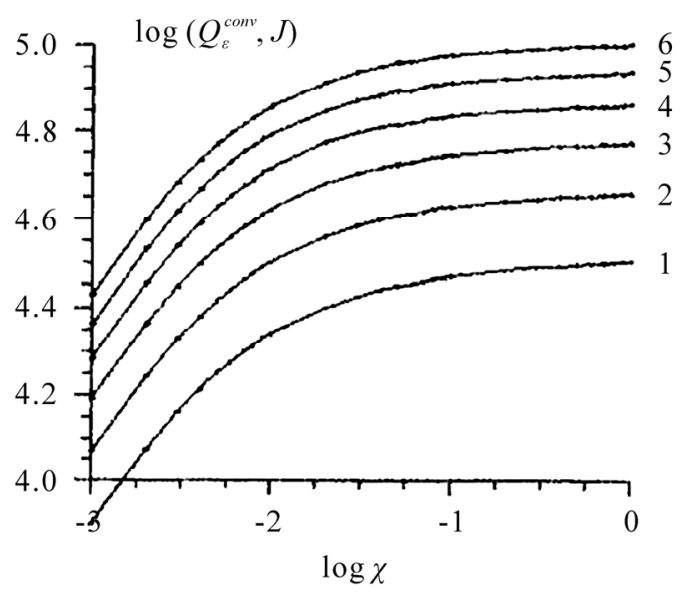

(c)

Figure 15. The graphics $Q_{\text {rad }}^{\text {conv }}, \Delta T_{\text {conv }}{ }^{*}, Q_{\varepsilon}{ }^{\text {conv }}=f(\chi) \quad$ (Quasi -equilibrium approximation) taken into account in calculations characteristics, then ones correspond with analogues for results of Figure13.

Figure 16 gives the quasi-equilibrium levels of radiate power $Q_{\text {rad }}^{\text {con }}$ ("a"), the differences $\Delta T_{\text {conv }}{ }^{*}$ between the ball lightning's translation temperatures and environment temperature ("b") as well as of the BL's energy resources component $Q_{\varepsilon}^{\text {conv }}$ ("c") but in the case, versus radius sphere of BL. The levels of others taken into account in calculations parameters correspond to conditions in calculation of Figure $14(\chi=0.01$, $V=4,6,8,10,12,14,16 \mathrm{~W} / \mathrm{A}$-plots $1-7$ respectively).

The heat transfer coefficient from environment to BL taken into account during forming of equations was determined on the base of criterion equation

$$
\begin{aligned}
N u & =\frac{2 \alpha_{\text {conv }} R_{b l}}{\lambda}=2+0.03 \operatorname{Pr}^{0.03} \operatorname{Re}^{0.54}+ \\
& +0.35 \operatorname{Pr}^{0.356} \operatorname{Re}^{0.58},
\end{aligned}
$$

being used for description of transfer heat between a sphere with radius $R_{b l}$ and flow running over with velocity $W$ [20]. With account of (32) and conformably to standard parameters of air the value of $\alpha_{\text {conv }}$ was concrete estimated by equation

$$
\begin{aligned}
\alpha_{\text {conv }}= & 2.581_{10^{-2}} \frac{1}{2 R_{b l}} \times \\
& \times\left(2+0.02 \operatorname{Re}^{0.54}+0.3080 \mathrm{Re}^{0.58}\right)
\end{aligned}
$$

Here:

$$
\operatorname{Re}=0.680_{10^{5}} W \bullet\left(2 R_{b l}\right)
$$

\subsection{Heat-Input with Mass-Exchange Mechanism}

For case of heat-input for energy feeding of BL in quasistationary state by mass-exchange mechanisms the energy input power was estimated by equation

$$
Q_{h i}{ }^{m t}=\pi R_{b l}^{2} W \rho_{\text {env }} c_{p}\left(T_{\text {env }}-T_{b l}\right)
$$

Here: $c_{p}, \rho_{e n v}, T_{e n v}$-density, heat capacity and temperature of environment accordingly;

$W$-velocity of environment flow relative of ball lightning.

It was taken into account that energy being inputted to BL with environment flow, in that case in connection with blowing off BL sphere by the environment flow, is used up at least in two ways: to compensate a energy loses of $\mathrm{BL}$ in connection with radiation, firstly, and to suppress cutting of energy states settlements (reproducetion of the ones) and practically to stabilize so, in conditions of continuous mass-exchange between the ball lightning and environment, the radiate capacity of BL as a whole (relation radiate capacity $\varepsilon_{g}$, effective radiate 


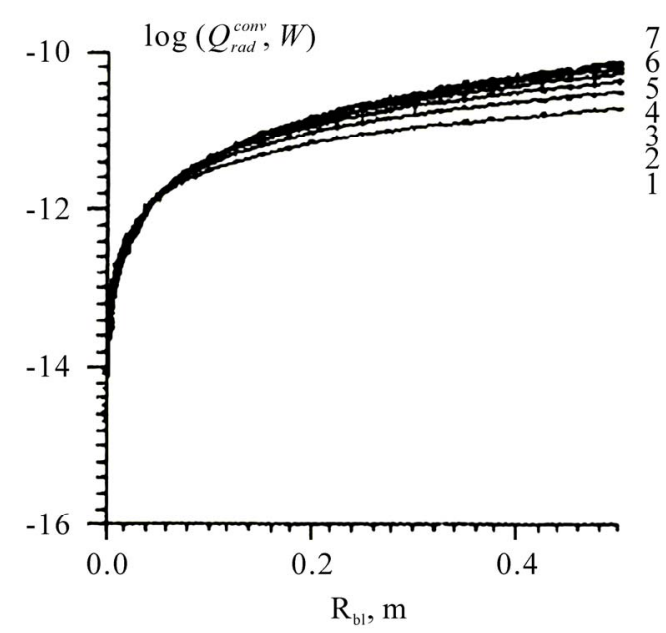

(a)

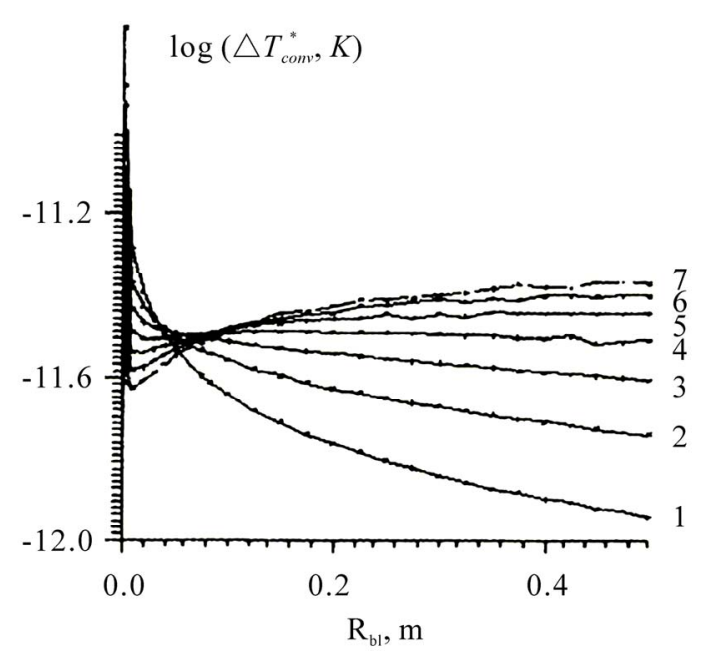

(b)

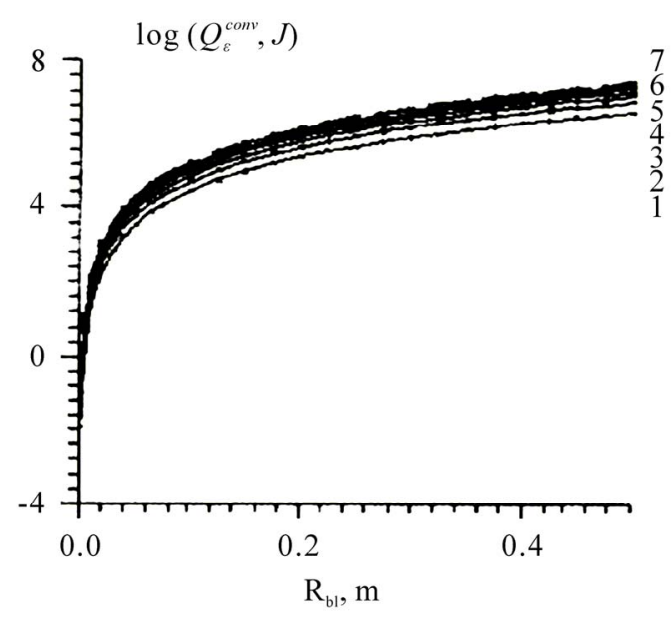

(c)

Figure 16. Relations $Q_{r a d}^{c o n v}, \Delta T_{\text {conv }}{ }^{*}, Q_{\varepsilon}{ }^{c o n v}=f\left(R_{b l}\right) \quad($ Quasiequilibrium approximation). temperature $T_{\text {rad }}$ and radiate power $Q_{\text {rad }}$.

Taking into consideration the common dependences specificity of excitation temperatures (see (5.6.2)) it is assumed that the BL radiate capacity is realized in that case by radiate transitions "on the distant approaches to equilibrium of BL-medium" ( $\left.T_{r a d}^{m t}=T_{e x c}{ }^{*}>\bar{T}_{e x c}{ }^{*}\right)$.

With estimations of radiation flow $Q_{\text {rad }}^{m t}$ by (25) and expenditure power onto reproduction of energy states settlements with

$$
\Delta Q_{h i}^{r e p}=V_{b l} \frac{S W}{V_{b l}} \varepsilon_{j}^{*} n_{j}^{*}=\pi R_{b l}{ }^{2} W \varepsilon_{j}{ }^{*} n_{j}^{*}
$$

the quasi-stationary conditions of BL were determined as

$$
\Delta T_{m t}{ }^{*}=T_{e n v}-T_{b l}=\frac{W \varepsilon_{j}{ }^{*} n_{j}^{*}+4 \varepsilon_{g} C_{0}\left(T_{r a d}^{m t} / 100\right)^{4}}{c_{p} \rho_{e n v} W} .
$$

Here: $n_{j}{ }^{*}$ - the settlement of level $\varepsilon_{j}{ }^{*}$ suitable to energy states excitation temperature; $S W / V_{b l}$-divisibility of BL-media mass-exchange.

In Figure 17 are shown logarithmic relationships of the power $Q_{\text {rad }}^{m t}$ ("a") radiated by ball lightning (with sphere radius $R_{b l}=0.075 \mathrm{~m}$ ), temperatures differences $\Delta T_{m t}{ }^{*}$ ("b") conforming to the power levels as well as the energy resources component $Q_{\varepsilon}^{m t}$ ("c") accumulated of BL-media in that case with connection of continuously reproduced deformation of energy states settlements distribution function versus relational dipoles keeping in BL-media composition.

The plots are built on the supposition that radiation is realized by transitions on the distant approaches to equilibrium of BL-media.

It was suggested that radiation temperature $T_{\text {rad }}^{m t}=5000 \mathrm{~K}$ and, in that case, corresponds to reproduced with connection of feeding the excitation temperature $T_{\text {exc }}{ }^{*}$ of BL-medium energy states with levels $\varepsilon_{j}{ }^{*}=6,8,10,12,14,16 \mathrm{eV}$ (curves 1-6 accordingly).

Taken into account quantity of environment temperature $T_{b l}=300 \mathrm{~K}$, of pressure-atmospheric one, of velocity blowing- $W=1 \mathrm{~m} / \mathrm{s}$.

To evaluate a quantity of $\psi$ the dependence

$$
\psi=\frac{\varepsilon_{j}^{*}}{e \Omega}\left(1-\frac{T_{b l}}{T_{r a d}^{m t}}\right)
$$

was used in that case.

It was taken into account that relational radiate degree $\varepsilon_{g}$ is determined with equation (27). At last, the $Q_{\varepsilon}^{m t}$ level was estimated by relationship

$$
Q_{\varepsilon}^{m t}=\varepsilon_{j}^{*} n_{j}^{*} V_{b l}=\frac{4}{3} \pi R_{b l}{ }^{3} \varepsilon_{j}^{*} \frac{\Delta n_{q}}{\psi}
$$

On Figure 18 the estimations relationships of the radiated power $Q_{\text {rad }}^{m t}$ ("a") and the temperatures differences 


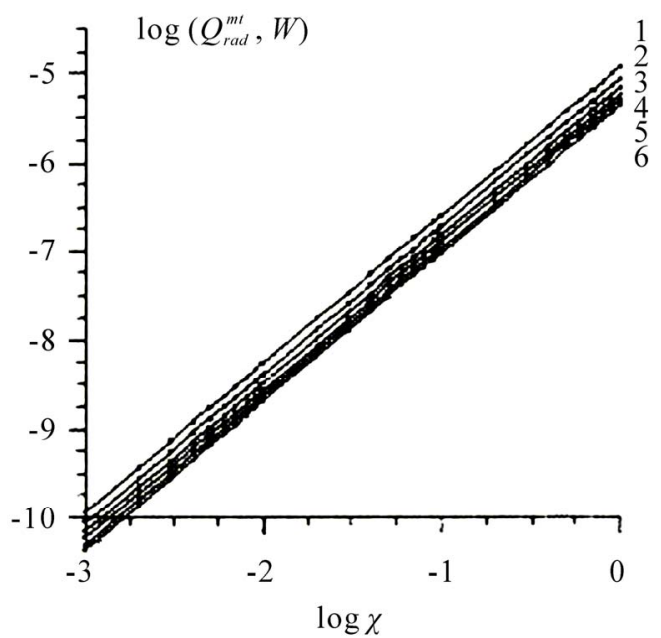

(a)

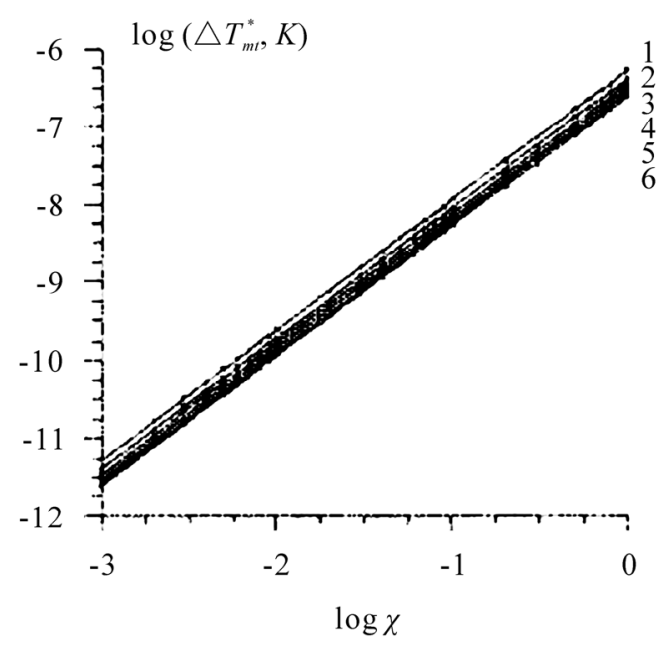

(b)

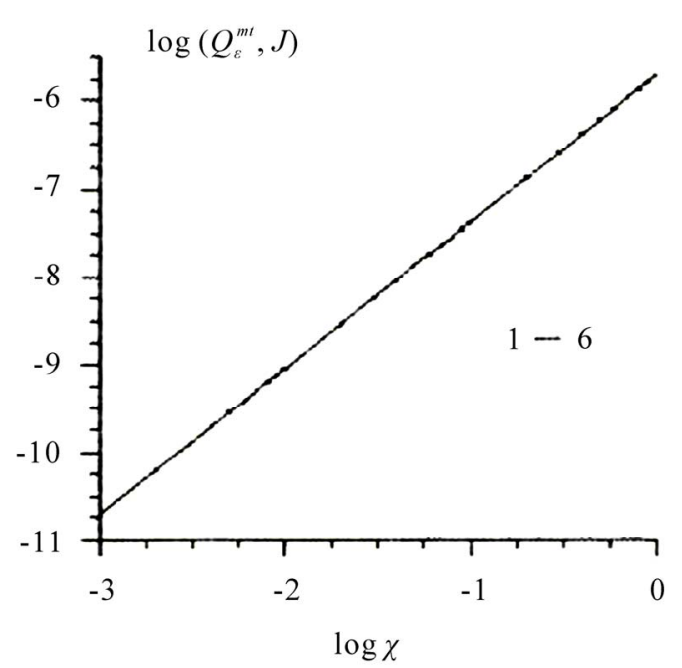

(c)

Figure 17. Relationships $Q_{r a d}^{m t}, \Delta T_{m t}{ }^{*}, Q_{\varepsilon}{ }^{m t}=f(\chi)$.

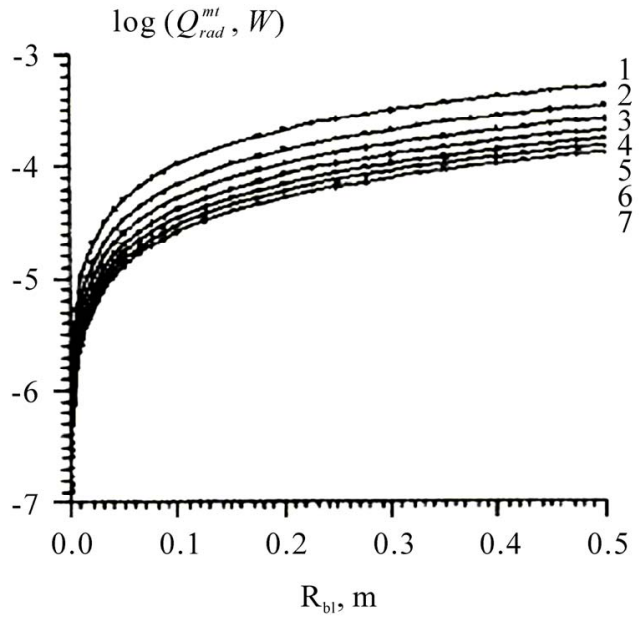

(a)

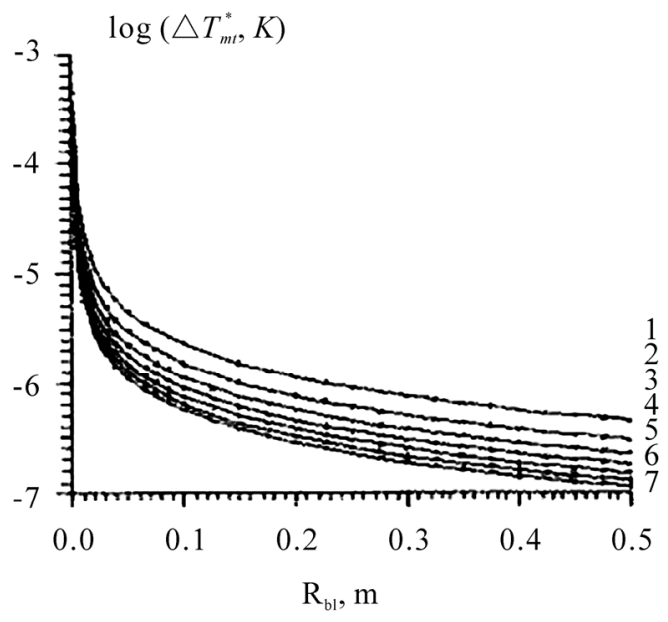

(b)

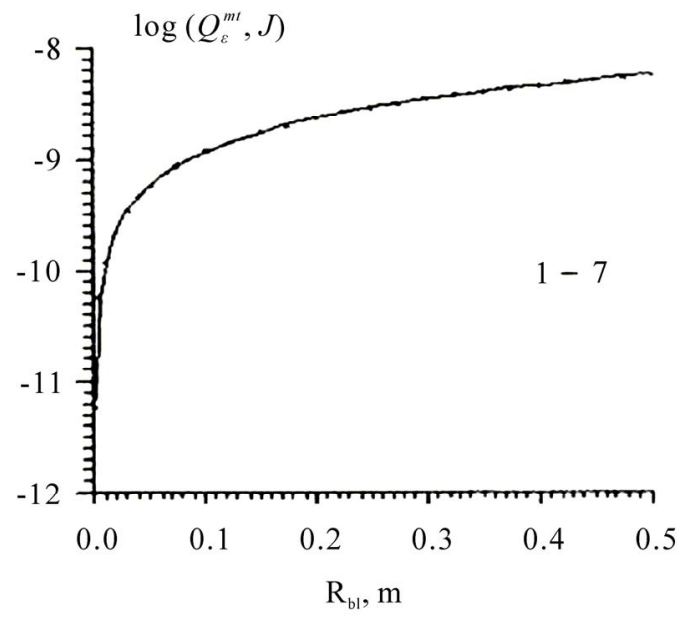

(c)

Figure 18. Relationships $Q_{r a d}^{m t}, \Delta T_{m t}{ }^{*}, Q_{\varepsilon}{ }^{m t}=f\left(R_{b l}\right)$ (Energy feeding by mass-transfer). 
$\Delta T_{m t}^{*}$ ("b") answering to being realized conditions along with energy $Q_{\varepsilon}^{m t}$ ("c") accumulated by BL-medium in connection with continuous reproduced deformation of energy states settlement distribution function versus sphere radius $R_{b l}$ of $\mathrm{BL}$ are presented.

The curves are being estimated by analogous kind in assumption that energy radiation by $\mathrm{BL}$ into environment is realized with radiate transitions on distant approaches to equilibrium of BL-media.

It was assumed that the radiate temperature $T_{\text {rad }}^{m t}=5000 \mathrm{~K}$ and corresponds to excitation temperature $T_{e x c}{ }^{*}$ of energy states with levels $\varepsilon_{j}{ }^{*}=4,6,8,10,12$, $14,16 \mathrm{eV}$ (plots 1-7 accordingly) under atmospheric pressure. Taken into account environment temperature level $T_{b l}=300 \mathrm{~K}$.

The dipole molecules proportion $\chi=0.01$ (dipole moment $d=1 D$ ). It was assumed also that the relative velocity of environment flow (BL blow-through) $W=1 \mathrm{~m} / \mathrm{s}$.

On Figure 19 the radiate power $Q_{\text {rad }}^{m t}$ of BL with sphere radius $R_{b l}=0.075 \mathrm{~m}$ and corresponding to levels of the one the temperatures differences $\Delta T_{m t}{ }^{*}$ as well as energy resources component $Q_{\varepsilon}^{m t}$ versus radiate temperature $T_{r a d}^{m t}$ of the ball lightning are presented.

The relationships are received by analogous with previous method and cover the diapason $T_{r a d}^{m t} \in(5000,50000) K$. Taken into account of the conventional BL-media composition corresponds to both relative dipoles content $\chi=0.001,0.005,0.01,0.05,0.1,0.5,1$ (the plots 1-7 correspondingly) and ionization potential $V=16 \mathrm{~W} / \mathrm{A}$ $\left(\varepsilon_{j}=16 \mathrm{eV}\right)$. As previous way it was assumed that $T_{b l}=300 \mathrm{~K}$, the pressure is atmospheric one, blowthrough velocity $W=1 \mathrm{~m} / \mathrm{s}$.

The analogous characteristics of BL with sphere radius $R_{b l}=0.075 \mathrm{~m}$ for different composition media ( $V=4,6,8,10,12,14,16 \mathrm{~W} / \mathrm{A}$, the curves 1-7 accordingly) with relative dipoles content $\chi=0.01$ are represented on Figure 20.

As in the above, the taken into account $T_{b l}=300 \mathrm{~K}$, pressure is the atmospheric one, blow velocity through BL $W=1 \mathrm{~m} / \mathrm{s}$. Taken into account the radiate temperature variation diapason in the case corresponds to $T_{\text {rad }}^{m t} \in(5000,100000) \mathrm{K}$.

Draw attention to the fact that levels of being estimated BL's energy characteristics (see Figures 11-20) are in gratifying agreement with characteristics of $\mathrm{BL}$ forecasted in the line of the notions about BL in the frame of the one's historically formed by observations portrait (see [15,16,19,21-23], for example).

It should be especially emphasized, in that number, that being formed so views about $\mathrm{BL}$ are whole consistent with results of experimental valuation of one's characteristics having been received by research workers of

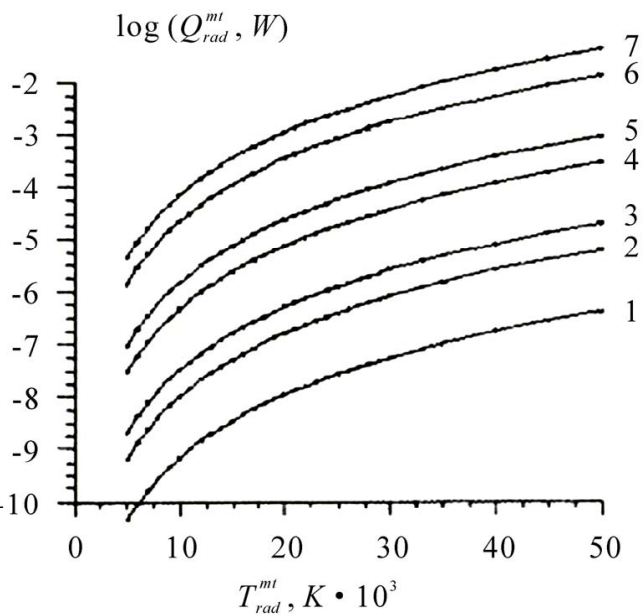

(c)

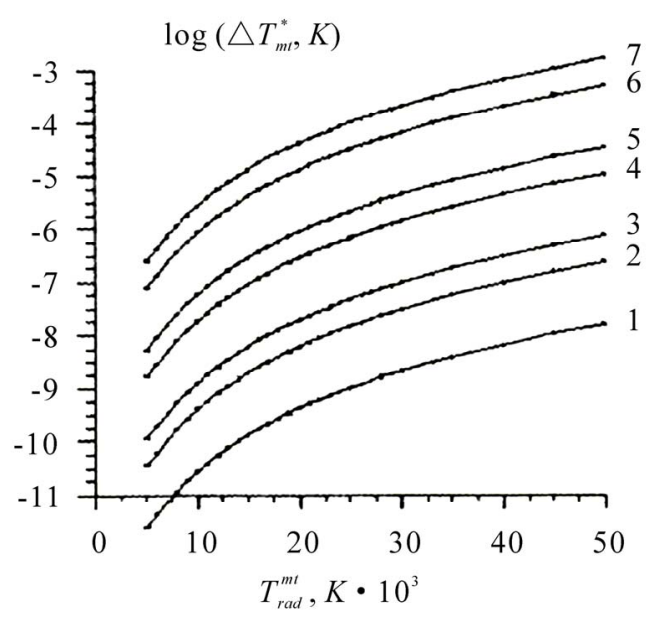

(c)

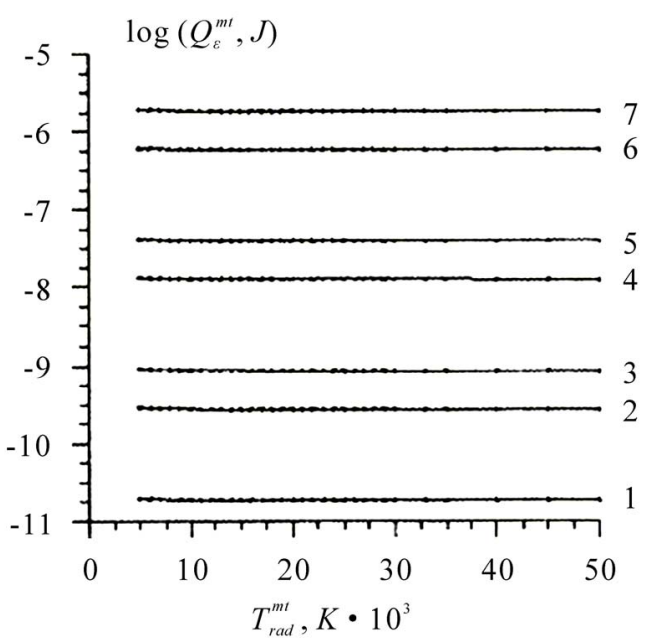

(c)

Figure 19. Relationships $Q_{r a d}^{m t}, \Delta T_{m t}{ }^{*}, Q_{\varepsilon}{ }^{m t}=f\left(T_{r a d}^{m t}\right)$. 


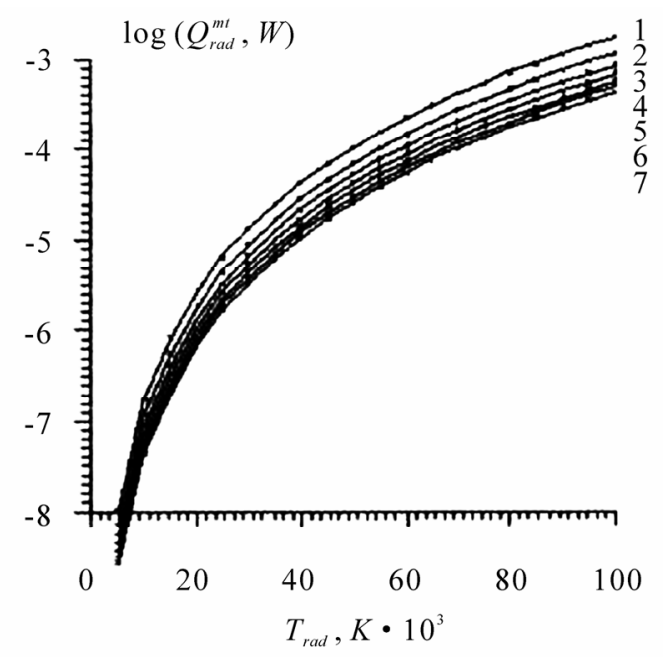

(a)

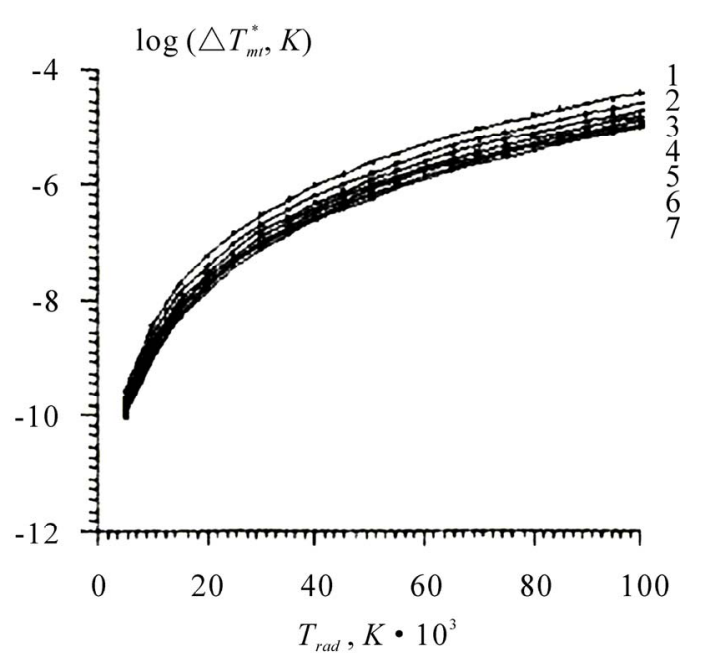

(b)

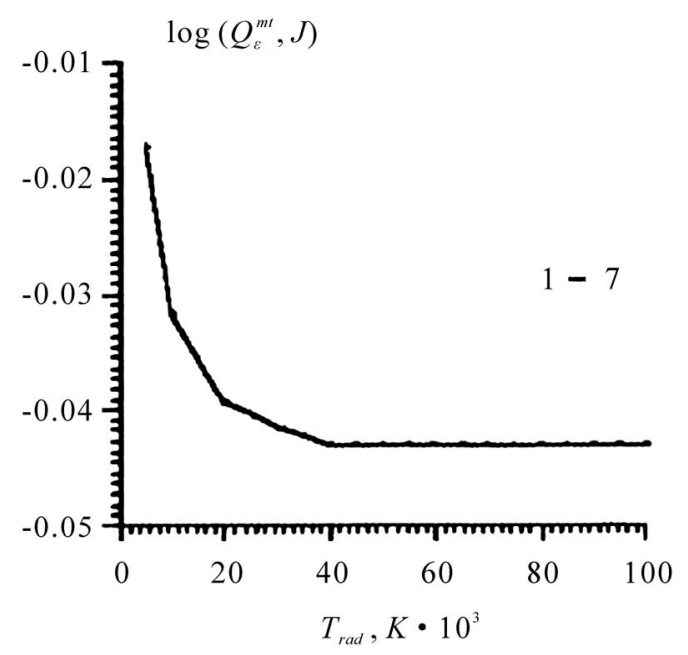

(c)

Figure 20. Relationships $Q_{r a d}^{m t}, \Delta T_{m t}{ }^{*}, Q_{\varepsilon}{ }^{m t}=f\left(T_{r a d}^{m t}\right)$.
Nuclear Physics Institute (Saint-Petersburg, Russia) [22, 23].

\subsection{Some Additional Words}

It is obvious that common conditions of origin along with existence of $\mathrm{BL}$ are characterized of electromagnetic disturbances that influence an high effect on environment. It can be said with confidence that, as one from other factors, occasional in origin electro-magnetic influences on atmosphere not only determine origin possibility of BL itself but in large degree substantiate movement itself of the one through space and one's common characteristics as well.

\section{Common Results}

By investigations results totality, upon overstepping the limits of generally accepted physical base, the conceptual views complex of ball lightning as about of special shape localized in the airspace material foundation with differences between one's translation temperature and excitation temperatures of one's chemical bonds and electron energy states have been formulated and is substantiated.

Adequacy and common rightfulness of the complex is jointly established by inclusion to examination in totality of all most significant characteristics and necessary existence conditions of BL, by revealed true of BL's characteristics being estimated on the complex base and of the ones being prognosticated in the frame of historically composed portrait of ball lightning and else by experimental confirmation of trustworthiness of the IEEC as of the physical base complex.

The complex is aimed to substantiation of jointly observed manifestations of BL. In the context of views being determined complex composition the localization possibility in airspace of ball lightning as material foundation with noted temperature differences (sphericity of BL) is confirmed. The nature of BL's radiation ability is based. The role of environment as source providing of BL with energy was revealed. Energy feeding mechanisms along with keeping conditions of quasi-stationary characteristics of BL are determined.

Formation channels of BL's energy resources (in that number of untraditional character ones) is placed. By top numerical estimations the energy resources levels are confirmed, possibilities and variation diapason of the ones (multi-faces of BL) are substantiated too.

Being received characteristics estimations of $\mathrm{BL}$ on the formulated complex base is found in satisfactory consent with characteristics of BL being prognosticated in the frame of the one's historically composed portrait.

Being revealed conformity like results of the work as a 
whole testify to in all common rightfulness of being developed point of view, further progress expediency of the one, possibilities to extend on the one's base our knowledge of nature.

Let us note else to independent scientific importance of proper IEEC, the ones' obvious fundamental character.

\section{Conclusions}

In the frame of an untraditional approach, upon overstepping the limits of generally accepted physical base the conceptual views complex about ball lightning as of quasi-stationary material foundation with differences between one's translation temperature and excitation temperatures of chemical bonds and electron energy states was formulated and are substantiated.

Every physical peculiarities of the base positions being used in the work present independent scientific interest. They note to existence of traditionally not accounted control channel of material media states in what electrical charge plays a role of control factor.

Jointly received result for the first time permits to formulate the scientific substantiated model of BL in such a way, for example.

Ball Lightning is containing of uncompensated (exceed) electrical charge atmospheric foundation with quasi-stationary in time characteristics and sizes, lifetime duration reaching several tens second, being displayed with energy radiation into environment in visual part specter too, manifesting else by an additional discharge of energy under disappearance, differing with capability to penetrate through inanes (hollows, cracks, splits) with essentially lesser sizes in respect to the own, to overcome so different barriers from dielectric materials (window-frame for example) and then to restore (reconstruct) in essence the ones' initial shape and characteristic, the foundations of properly forming, existence and charac-teristics of that under interaction with environment jointly are provided with occasional in origin electro- magnetic disturbances of atmosphere, presence in the one's composition of dipole components (of components with unequal to zero dipole moment) along with de-pendence of Internal Energy Equilibrium Conditions of material media from presence or absence uncompensated electrical charge in the ones' composition.

Being developed in this work approach is of an universal character, is grounded upon overstepping the limits of generally accepted physical base and presents itself to be perspective for solving of not only of BL-problem but and of wide circle of scientific and is applied problems. The last, however, demands of confirmation with additional purposeful researches.

\section{As Concluding Remarks}

In the context of views, being jointly formed by [1-13] and this work, definite interest presents a gaze to problem of combustion in detonation (DC) that, in connection with having being multi-verified by experiment but not interpreted just till now anomalously high heat release of DC, draws to itself fixed, constantly increasing acrossthe-board attention.

On being estimate an expedient decision way of this problem an attention should be drawn to the fact that DC as process is developed above all on background of external acoustic disturbances. With regard to [17] so, combustion in detonation is realized in conditions that are the most convenient for generation of macroscopic AQN-formations in combustion products medium and, hence, for excitement in the ones' compound, at the relaxation stage to IEEC, of reactions, among them, may be one or up intra-nuclear, energy producing (exothermic) reactions too.

To put it differently, peculiar to DG high levels of heat release reveal not only ones' consistency to notions of both $[1-13,17]$ and this one, but even just with fact proper of ones' displaying (unquestionable having been confirmed by numerous observations) testify to validity of the IEEC in themselves as well as of conceptual notions of being referred above works as a whole.

\section{References}

[1] V. A. Pinchuk, "The Nature and Energy Sources of Ball Lightning," Proceedings of 24th International Electric Propulsion Conference/Paper IEPC-95-233, Moscow, 19 -23 September 1995, pp. 1-11.

[2] V. A. Pinchuk, "Physical Basis of Cold Fusion and Prospects of its Application as Engine Power Source," Proceedings of the 3rd International Conference on New Energy Systems and Conversions, Kazan, Russia, 8-13 September 1997, pp. 189-192.

[3] V. A. Pinchuk, "The Phenomenon of Ball Lightning and Cold Nuclear Synthesis," Proceedings of the 2nd International Symposium on Energy, Environment and Economic, Kazan, Russia, 7-10 September 1998, Vol. 2. pp. 199 -213 .

[4] V. A. Pinchuk, "Reality and the Nature of Cold Nuclear Synthesis are Justified by the Ball Lightning Existence," Proceedings of the 4th International Conference on New Energy Systems and Conversions, Osaka, Japan, 27-30 June 1999, pp. 361-368.

[5] V. A. Pinchuk, "The Common Techniques Features and Prospects of Cold Nuclear Synthesis Assimilation to Needs of Power Engineering," Proceedings of the 5th International Conference on New Energy Systems and Conversions, Shanghai, P.R. China, 22-25 August 2001, pp. 369-374. 
[6] V. A. Pinchuk, "Physics of Material Formations with Anomalous Characteristics," Combustion \& Plasmochemistry, Vol. 2, No. 2, 2004, pp. 81-100 (in Russian).

[7] V. A. Pinchuk, "The Physics of Material Formations with Anomalous Characteristics," Proceedings " $\mathrm{htm}$ " of International Conference on Exploring Innovation in Education and Research, Tainan, Taiwan, 1-5 March 2005, Paper W38, pp. 1-15.

[8] V. A. Pinchuk, "Once More about Anomalies Physics," Proceedings of International Conference on Engineering Education, Gliwice, Poland, 25-29 July 2005, Vol. 2. pp. 800-814.

[9] A. V. Pinchuk and V. A. Pinchuk, "Chargeous Channel of Reactions Excitation on Burning," Combustion \& Plasmo-chemistry, Vol. 5, No. 4, 2007, pp. 307-319 (in Russian).

[10] A. V. Pinchuk and V. A. Pinchuk, "Conception: Mutation of Material Media Compositions as Process Being Exited with Chargeous Influences," RSA Trudi Academenergo, No. 2, 2008, pp. 101-113 (in Russian).

[11] A. V. Pinchuk and V. A. Pinchuk, "Regarding Mutation of Media's Compounds Mutation as of Process Being Excited by Chargeous Influences," Bulletin of BSTU, 2008, No. 2, pp. 50-59 (in Russian).

[12] A. V. Pinchuk and V. A. Pinchuk, "The Formations with Surplus Charge: Nature and Influence upon Burning Process Stability," 6th International Seminar on Flame Structure. Book of Abstracts, Brussels, Belgium, 2008, p. 60.

[13] A. V. Pinchuk, "Conditions of Internal Energy Equilibrium as Experimental Researches Object," In: A. F. Utkin, ed., Proceedings of International Scientific Technical
Conference "4th Utkinskie Chtenija" (Saint-Petersburg, 21-23 May 2009), Saint-Petersburg, BSTU, Vol. 2, 2009, pp. 6-9 (in Russian).

[14] A. I. Grigor'ev, I. D. Grigor'eva and S. O. Shirjaeva, "About Ball Lightning Stable in Relation to Own Uncompensated Charge," Journal of Technical Physics, Vol 65, No. 2, 1995, pp.1-10 (in Russian).

[15] S. Singer, "Ball Lightning Nature," Moscow: MIR, 1973, p. 320. (in Russian).

[16] G. Barry. "Ball and Line Lightning," Moscow: MIR, 1983, p. 285 (in Russian).

[17] V. A. Pinchuk, "Low-Temperature Plasma to External Acoustic Disturbances," Journal of Engineering Physics and Thermophysics (JEPTER), Vol. 67, No. 1-2, 1994, pp. 781-784.

[18] I. S. Borovikov, "About Minimum of Entropy Working Out," Journal of Engineering Physics and Thermophysics, Vol. 35, No. 3, 1978, pp. 415-419 (in Russian).

[19] B. M. Smirnov, "Ball Lightning Nature," UFN, Vol. 116, No. 4, 1975, p. 731 (in Russian).

[20] S. S. Kutateladze, "Base of Heat Transfer," Moscow-Leningrad Mashgiz, 1957, p. 383 (in Russian).

[21] I. P. Stakhanov, "Ball Lightning's Physical Nature," Moscow: Atomizdat, 1979 (in Russian)

[22] G. D. Shabanov and B. Yu. Sokolovski, "Macroscopic Division of Charge in Impulse Electrical Discharge," Plasma Physics, Vol. 31, No. 6, 2005, pp. 560-566 (in Russian).

[23] G. D. Shabanov, "Lightning on the Table," Science and Life, No. 11, 2009, pp. 38-43 (in Russian). 


\section{Nomenclatures}

e elementary charge (C);

$g \quad$ statistical state weight;

$h \quad$ Plank's constant $(\mathrm{J} \cdot \mathrm{s})$;

$k \quad$ Boltsman's constant $(\mathrm{J} / \mathrm{K})$;

$d \quad$ dipole moment $(\mathrm{C} \cdot \mathrm{m})$;

$\Phi \quad$ thermodynamic potential $\left(\mathrm{J} / \mathrm{m}^{3}\right)$;

$\mu \quad$ chemical potential $(\mathrm{J} / \mathrm{kmole})$;

$q \quad$ electrical charge, $(\mathrm{C})$;

$C \quad$ electrical capacity (C/V);

$c_{p} \quad$ specific heat capacity $(\mathrm{J} /(\mathrm{kg} \cdot \mathrm{K}))$;

$Q \quad$ energy $(\mathrm{J})$, power $(\mathrm{J} / \mathrm{s})$;

$R \quad$ radius $(\mathrm{m})$, universal gas constant $\mathrm{J} /($ mole $\cdot \mathrm{K}))$;

$N \quad$ molar concentration $\left(\mathrm{mole} / \mathrm{m}^{3}\right)$;

$S \quad$ area $\left(\mathrm{m}^{2}\right)$;

$n \quad$ species concentration $\left(\mathrm{m}^{-3}\right)$;

$\alpha \quad$ ionization degree, heat transmission coefficient $\left(\mathrm{W} /\left(\mathrm{m}^{2} \cdot \mathrm{K}\right)\right)$;

$\psi \quad$ relative keeping of surplus electron component;

$P, p \quad$ pressure $\left(\mathrm{kg} /\left(\mathrm{m} \cdot \mathrm{s}^{2}\right)\right)$;

$T \quad$ temperature $(\mathrm{K})$;

$\rho \quad$ density $\left(\mathrm{kg} / \mathrm{m}^{3}\right)$;

$\chi \quad$ relative keeping of dipole component; $\varepsilon_{0} \quad$ electrical constant $(\mathrm{C} /(\mathrm{V} \cdot \mathrm{m}))$;

$C_{0} \quad$ radiate ability coefficient of absolute black surface $(\mathrm{W} /(\mathrm{m} 2 \cdot \mathrm{K} 4))$;

$\varepsilon_{g} \quad$ relative blackness degree;

$\Omega \quad$ electrical potential (V);

$V \quad$ volume $\left(\mathrm{m}^{3}\right)$; ionization potential $(\mathrm{V})$;

$W \quad$ velocity $(\mathrm{m} / \mathrm{s})$.

\section{Symbol}

$0 \quad$ undisturbed parameter;

a neutral;

e electron;

$i$ ion;

c capacitor;

conv convection;

$m t \quad$ mass transfer;

sf safe;

st safe tension;

rad radiation;

hi heat input;

bl ball lightning;

env environment;

exc excited parameter,

“." true to equilibrium 